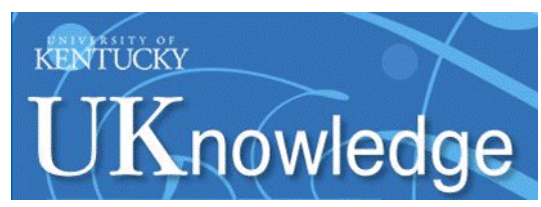

University of Kentucky

UKnowledge

\title{
Expanded Iron UTA Spectra-Probing the Thermal Stability Limits in AGN Clouds
}

\author{
Gary J. Ferland \\ University of Kentucky, gary@uky.edu \\ R. Kisielius \\ Vilnius University, Lithuania \\ F. P. Keenan \\ Queen's University Belfast, UK \\ P. A. M. van Hoof \\ Royal Observatory of Belgium, Belgium \\ V. Jonauskas \\ Vilnius University, Lithuania
}

See next page for additional authors

Follow this and additional works at: https://uknowledge.uky.edu/physastron_facpub

Part of the Astrophysics and Astronomy Commons, and the Physics Commons

Right click to open a feedback form in a new tab to let us know how this document benefits you.

\section{Repository Citation}

Ferland, Gary J.; Kisielius, R.; Keenan, F. P.; van Hoof, P. A. M.; Jonauskas, V.; Lykins, Matt L.; Porter, R. L.; and Williams, R. J. R., "Expanded Iron UTA Spectra-Probing the Thermal Stability Limits in AGN Clouds" (2013). Physics and Astronomy Faculty Publications. 62.

https://uknowledge.uky.edu/physastron_facpub/62

This Article is brought to you for free and open access by the Physics and Astronomy at UKnowledge. It has been accepted for inclusion in Physics and Astronomy Faculty Publications by an authorized administrator of UKnowledge. For more information, please contact UKnowledge@lsv.uky.edu. 


\section{Expanded Iron UTA Spectra-Probing the Thermal Stability Limits in AGN Clouds}

\section{Digital Object Identifier (DOI)}

https://doi.org/10.1088/0004-637X/767/2/123

\section{Notes/Citation Information}

Published in The Astrophysical Journal, v. 767, no. 2, 123, p. 1-12.

(c) 2013. The American Astronomical Society. All rights reserved. Printed in the U.S.A.

The copyright holder has granted permission for posting the article here.

\section{Authors}

Gary J. Ferland, R. Kisielius, F. P. Keenan, P. A. M. van Hoof, V. Jonauskas, Matt L. Lykins, R. L. Porter, and R. J. R. Williams 


\title{
EXPANDED IRON UTA SPECTRA-PROBING THE THERMAL STABILITY LIMITS IN AGN CLOUDS
}

\author{
G. J. Ferland ${ }^{1}$, R. Kisielius ${ }^{2}$, F. P. Keenan ${ }^{3}$, P. A. M. van Hoof ${ }^{4}$, V. Jonauskas ${ }^{2}$, M. L. Lykins ${ }^{1}$, \\ R. L. PORTER ${ }^{5}$, AND R. J. R. Williams ${ }^{6}$ \\ ${ }^{1}$ Department of Physics and Astronomy, University of Kentucky, Lexington, KY 40506, USA; gary @ pa.uky.edu \\ 2 Institute of Theoretical Physics and Astronomy, Vilnius University, A. Goštauto 12, LT-01108, Lithuania \\ ${ }^{3}$ Astrophysics Research Centre, School of Mathematics and Physics, Queen's University Belfast, Belfast BT7 1NN, UK \\ ${ }^{4}$ Royal Observatory of Belgium, Ringlaan 3, B-1180 Brussel, Belgium \\ ${ }^{5}$ Department of Physics, University of Georgia, Athens, GA 30602, USA \\ ${ }^{6}$ AWE plc, Aldermaston, Reading RG7 4PR, UK \\ Received 2012 November 4; accepted 2013 February 28; published 2013 April 4
}

\begin{abstract}
The Fe unresolved transition arrays (UTAs) produce prominent features in the $\sim 15-17 \AA$ wavelength range in the spectra of active galactic nuclei (AGNs). Here, we present new calculations of the energies and oscillator strengths of inner-shell lines from Fe XIV, Fe Xv, and Fe XVI. These are crucial ions since they are dominant at inflection points in the gas thermal stability curve, and UTA excitation followed by autoionization is an important ionization mechanism for these species. We incorporate these, and data reported in previous papers, into the plasma simulation code Cloudy. This updated physics is subsequently employed to reconsider the thermally stable phases in absorbing media in AGNs. We show how the absorption profile of the Fe xIV UTA depends on density, due to the changing populations of levels within the ground configuration.
\end{abstract}

Key words: atomic data - atomic processes - galaxies: active - line: formation - methods: numerical - X-rays: galaxies

Online-only material: color figures, machine-readable tables

\section{INTRODUCTION}

X-ray observations of active galactic nuclei (AGNs) have revealed the existence of a broad absorption feature in the $15-17 \AA$ wavelength range. This feature arises from an unresolved transition array (UTA) due primarily to $2 p \rightarrow 3 d$ inner-shell absorption lines in iron ions with an open $\mathrm{M}$ shell (Fe I-Fe XVI), and was first identified by Sako et al. (2001) in the XMM-Newton spectrum of the quasar IRAS $13349+2438$. Since then, the UTA has been detected in numerous AGN (see, for example, Longinotti et al. 2010; Lestinsky et al. 2009, and references therein). It is believed to arise in the warm, relatively low ionization, absorbing gas surrounding the central supermassive black hole in the AGN.

Behar et al. (2001) first noted the diagnostic potential of the UTA, and showed that their central wavelengths, absorption profiles, and equivalent widths can provide information on the ionization structure, kinematics, and column densities of the warm absorbing material in the AGN. These authors also produced atomic data required for the modeling of the UTA, including energy levels (wavelengths) and oscillator strengths for the 16 iron charge states Fe I through Fe XVI, calculated with the multiconfiguration, relativistic HULLAC computer code (Bar-Shalom et al. 2001). Since then, several authors have calculated wavelengths and oscillator strengths for the UTA. These include Kisielius et al. (2003), who produced atomic data for Fe XV and Fe XVI using the configuration interaction code cIv3 (Hibbert 1975) with the inclusion of relativistic effects by adding Breit-Pauli operators to the Hamiltonian (Hibbert et al. 1991). More recently, Gu et al. (2006) calculated results for Fe VI through Fe XVI employing second-order manybody perturbation theory (MBPT; Lingren 1974). Furthermore, Beiersdorfer et al. (2012) used the relativistic multi-reference Møller-Plesset perturbation theory to calculate the energy levels of Fe XVI, including those of the autoionizing levels with a hole state in the L shell. They obtained very good agreement between the calculated L-shell transition wavelengths and those from recent laboratory measurements.

Here, we present extended calculations of UTA energies and radiative transition rates for Fe XIV, Fe XV, and Fe XVI. These are combined with other improvements in the plasma simulation code Cloudy (Ferland et al. 2013) to generate a range of models of absorbing clouds in AGN. Calculations were performed with r6433 of the Cloudy development branch, and will be part of the 2013 release of the code. We also perform a thermal stability analysis to identify the thermally stable phases where clouds can exist, and show that Fe XIV, Fe Xv, and Fe XvI probe the high ionization end of the warm stable phase.

\section{NEW ATOMIC DATA FOR Fe XIV, Fe Xv, AND Fe XVI TRANSITIONS}

Previously, a comparatively small set of inner-shell photoexcitation data for $\mathrm{Fe}$ XV and Fe XVI was reported by Kisielius et al. (2003). In that work energy levels were identified, and electric dipole transition wavelengths, oscillator strengths, and transition probabilities determined. Specifically, transitions involving photoexcitation from the inner $2 p$ shell to the outer $n=3$ shell were investigated using the relativistic Breit-Pauli approach, and an assessment of the accuracy of the derived atomic data was given. In the present work, the data set has been significantly extended. First of all, we add line data involving the transitions from the $2 s$ shell since their wavelengths lie within the same UTA range. Furthermore, we determine excitation (both from $2 s$ and $2 p$ shells) to the $n=3,4$, and 5 shells. Finally, photoexcitation data for the same type of transitions in Fe XIV ions are derived.

In our current work, we use the configuration interaction (CI) approximation for atomic calculations. Particularly, the wellestablished computer code CIV3 of (Hibbert 1975) is exploited to 
determine multiconfiguration eigenfunctions and eigenvalues of considered energy levels. After this initial step, we derive atomic data such as electric dipole (E1) radiative transition wavelengths $\lambda$, oscillator strengths $f$, and transition probabilities (rates) $A$ for the transitions from inner $n=2$ shell of Fe XIV, Fe XV, and Fe XVI ions. The adopted CI method is suitable for production of very accurate data, where the correlation effects play a vital role in theoretical data accuracy.

Data are generated in the relativistic Breit-Pauli approach (Hibbert et al. 1991) for all lines arising from $2 s$ and $2 p$ shell electron transitions to the $n=3,4$, and 5 shells. Combining these two approaches, we can reliably determine $L S J$-energy levels and, consequently, the E1 transition data for fine-structure levels. When CI wave functions for the fine-structure levels of the ground and excited states are determined, the electric dipole transition absorption oscillator strength $f$ is given by

$$
f=2 / 3 g_{i}^{-1} \Delta E_{i j} S^{2},
$$

where $g_{i}=2 J_{i}+1$ is the statistical weight of the initial (ground) level, $\Delta E_{i j}$ is the energy difference between the upper and lower level, and $S$ is transition line strength (matrix element). The corresponding electric dipole line emission transition probability $A$ (in $\mathrm{s}^{-1}$ ) is given by

$$
A=2.142 \times 10^{10} g_{j}^{-1}\left(\Delta E_{i j}\right)^{3} S^{2},
$$

where $g_{j}=2 J_{j}+1$ is the statistical weight of the excited-state level $j$.

A more detailed description of the approximations used in the present work may be found in Kisielius et al. (2003), where inner $2 p$ shell photoexcitation was considered for Fe XV and Fe XVI. The approach applied in the current paper for a much wider set of lines and Fe ions is very similar. Kisielius et al. (2003) have considered in detail the accuracy of determined results by applying mainly the internal indicators such as convergence of transition wavelengths with increasing the size of CI wave function expansion or agreement between length and velocity forms of oscillator strength values. They have estimated that $2 p-3 l$ transition wavelength accuracy was $0.2 \%$ whereas oscillator strength accuracy was assessed as $10 \%$ for the lines having $f \geqslant 5 \times 10^{-5}$. The conclusions on the accuracy of the atomic data made by Kisielius et al. (2003) are also applicable to the present results.

\subsection{Fe XVI Lines}

The ground level of the $\mathrm{Fe}^{15+}$ ion is $1 s^{2} 2 s^{2} 2 p^{6} 3 s^{2} S_{1 / 2}^{\mathrm{e}}$. Its CI wave function expansion was chosen to include no more than two electrons excited from the $2 p$ and $3 s$ shells in the case of the lines arising from the $2 p$ shell, and no more than two electrons excited from $2 s$ and $3 s$ shells for the lines arising from the $2 s$ shell. The significant difference between the calculation of $n=3$ and 4 lines lies in the fact that we use correlation radial orbitals $\overline{4} s, \overline{4} p, \overline{4} d, \overline{4} f$ in the former case to improve the calculated wavelength accuracy (for more details see Kisielius et al. 2003). In the latter case, these orbitals represent real states while the configuration sets remain the same.

\subsubsection{Excitation from the $2 p$ Shell}

Following this approach, the ground state CI wave function expansion for the transition arrays $2 p-3 l$ and $2 p-4 l$ was the same and included $2 p^{6} 3 l, 2 p^{6} 4 l(l=0,2), 2 p^{5} 3 s 3 p$,
$2 p^{5} 3 p 3 d, 2 p^{5} 3 l 4 l^{\prime}, 2 p^{5} 4 l 4 l^{\prime}\left(l=0,1,2 ; l^{\prime}=0,1,2,3\right)$ evenparity configurations, coupled to the ${ }^{2} S,{ }^{2} P,{ }^{4} P,{ }^{4} D L S$-terms. This CI expansion generates $94 L S J$-levels with total orbital quantum number $J=1 / 2$. Due to selection rules for E1 transitions, the excited configuration with the vacancy in the $2 p$ shell can only have levels with $J=1 / 2$ and $J=3 / 2$. We use a CI wave function expansion consisting of odd-parity configurations $2 p^{6} 3 p, 2 p^{6} 4 p, 2 p^{5} 3 l^{2}(l=0,1,2), 2 p^{5} 3 s 3 d$, $2 p^{5} 3 l 4 l^{\prime}\left(l=0,1,2 ; l^{\prime}=0,1,2,3\right), 2 p^{5} 4 l^{2}(l=0,1,2,3)$, $2 p^{5} 4 l 4 l^{\prime}\left(l=0,1 ; l^{\prime}=2,3\right)$ coupled to the ${ }^{2} S,{ }^{2} P,{ }^{4} P,{ }^{4} D L S$ terms for $J=1 / 2$ and to the ${ }^{4} S,{ }^{2} P,{ }^{4} P,{ }^{2} D,{ }^{4} D,{ }^{4} F$ terms for $J=3 / 2$. Consequently, we consider 93 levels with $J=1 / 2$ and 147 levels with $J=3 / 2$ for the excited configurations having electrons in the outer $n=3$ and 4 shells and the vacancy in the inner $2 p$ shell.

Very similar configuration sets were used to obtain atomic data for the transitions from the $2 p$ shell to the outer $5 l$ shell. The ground state wave function expansion included $2 p^{6} n l$ $(n=3,4,5 ; l=0,2), 2 p^{5} 3 s 3 p, 2 p^{5} 3 p 3 d, 2 p^{5} 3 l 5 l^{\prime} \quad(l=$ $\left.0,1,2 ; l^{\prime}=0,1,2,3\right), 2 p^{5} 5 l 5 l^{\prime}\left(l=0,1,2,3 ; l^{\prime}=0,1,2,3\right)$ even-parity configurations coupled to the ${ }^{2} S,{ }^{2} P,{ }^{4} P,{ }^{4} D L S$ terms. This yields $95 L S J$-levels having $J=1 / 2$.

The same (as in $n=3,4$ case) terms for the excited configuration levels with $J=1 / 2$ and $J=3 / 2$ were considered, while the CI expansion included configurations $2 p^{6} n p(n=3,4,5)$, $2 p^{5} 3 l^{2}(l=0,1,2), 2 p^{5} 3 s 3 d, 2 p^{5} 3 l 5 l^{\prime}\left(l=0,1,2 ; l^{\prime}=\right.$ $0,1,2,3) 2 p^{5} 5 l^{2}(l=0,1,2,3), 2 p^{5} 5 s 5 d, 2 p^{5} 5 p 5 f$. For $J=1 / 2$, the CI expansion included 94 levels whereas for $J=3 / 2$ the number was 148 . Levels arising from the configurations with $5 g$ electrons were not included in the CI expansion because such lines are very weak compared to other $n=5$ transitions.

\subsubsection{Excitation from the $2 s$ Shell}

A similar approach for the configuration sets to that adopted for the $2 p$ shell was taken when calculating atomic data for the lines corresponding to the transitions from the inner $2 s$ shell to the outer $n=3,4$, and 5 shells. For the $n=$ 3 and 4 lines, the ground state CI expansion included 56 levels with $J=1 / 2$ from the configurations $2 p^{6} n l(n=$ $3,4 ; l=0,2) 2 p^{5} 3 s 3 p, 2 p^{5} 3 p 3 d, 2 s 2 p^{6} 3 l^{2}(l=0,1,2)$, $2 s 2 p^{6} 3 l 4 l^{\prime}\left(l=0,1,2 ; l^{\prime}=0,1,2,3\right), 2 s 2 p^{6} 4 l^{2}, \quad(l=$ $0,1,2,3), 2 s 2 p^{6} 4 s 4 d, 2 s 2 p^{6} 4 p 4 f$ coupled to the ${ }^{2} S,{ }^{2} P$, ${ }^{4} P,{ }^{4} D$ terms. The upper-state configuration set consisted of $2 p^{6} 3 p, 2 p^{6} 4 p, 2 p^{6} 4 f, 2 s 2 p^{6} 3 s 3 p, 2 s 2 p^{6} 3 p 3 d, 2 s 2 p^{6} n l 4 l^{\prime}$ ( $\left.n=3,4 ; l=0,1,2 ; l^{\prime}=0,1,2,3\right)$ configurations coupled to the terms ${ }^{2} S,{ }^{2} P,{ }^{4} P,{ }^{4} D,{ }^{6} D,{ }^{6} F$ for the $J=1 / 2$ levels, and to the ${ }^{2} P,{ }^{2} D,{ }^{4} S,{ }^{4} P,{ }^{4} D,{ }^{4} F,{ }^{6} P,{ }^{6} D,{ }^{6} F,{ }^{6} G$ terms for $J=3 / 2$. This selection produced 38 and 58 levels for $J=1 / 2$ and $3 / 2$, respectively.

For the lines corresponding to the transitions from the inner $2 s$ shell to the outer $n=5$ shell, the ground state wave function CI expansion included configurations $2 p^{6} n l(n=3,4,5 ; l=$ $0,2), 2 p^{5} 3 s 3 p, 2 p^{5} 3 p 3 d, 2 s 2 p^{6} 3 l^{2}(l=0,1,2), 2 s 2 p^{6} 3 s 3 d$, $2 s 2 p^{6} 3 l 5 l^{\prime}\left(l=0,1,2 ; l^{\prime}=0,1,2,3\right), 2 s 2 p^{6} 5 l^{2}(l=0,1,2)$, $2 s 2 p^{6} 5 s 5 d, 2 s 2 p^{6} 5 p 5 f$ coupled to the terms ${ }^{2} S,{ }^{2} P,{ }^{4} P,{ }^{4} D$. This generates a total of 57 levels with $J=1 / 2$. For the upper state configuration set, we included configurations $2 p^{6} n l$ $(n=3,4,5 ; l=1,3), 2 s 2 p^{6} 3 s 3 p, 2 s 2 p^{6} 3 p 3 d, 2 s 2 p^{6} n l 5 l^{\prime}$, ( $\left.n=3,4 ; l=0,1,2 ; l^{\prime}=0,1,2,3\right)$ which were coupled to the terms ${ }^{2} S,{ }^{2} P,{ }^{4} P,{ }^{4} D,{ }^{6} D,{ }^{6} F$, producing 39 levels with $J=1 / 2$. For $J=3 / 2$, we considered the same terms as in the $n=4$ lines case, producing 59 levels. 
Table 1

Transitions $2 p-n_{j} d$ and $2 s-n_{j} p$ from the L Shell to $n_{j}=3,4$, and 5 Shells in $\mathrm{Fe}^{15+}$

\begin{tabular}{lccccc}
\hline \hline$n_{i} l_{i}$ & $n_{j}$ & $g_{i}$ & $g_{j}$ & $\lambda(\AA)$ & $f_{i j}$ \\
\hline $2 \mathrm{p}$ & 3 & 2 & 4 & 15.153 & 0.841 \\
$2 \mathrm{p}$ & 3 & 2 & 2 & 15.083 & 0.795 \\
$2 \mathrm{p}$ & 3 & 2 & 4 & 14.996 & 0.697 \\
$2 \mathrm{~s}$ & 3 & 2 & 4 & 13.951 & 0.291 \\
$2 \mathrm{p}$ & 4 & 2 & 4 & 12.336 & 0.230 \\
$2 \mathrm{p}$ & 4 & 2 & 4 & 12.469 & 0.227 \\
$2 \mathrm{p}$ & 3 & 2 & 4 & 15.408 & 0.158 \\
$2 \mathrm{p}$ & 4 & 2 & 2 & 12.330 & 0.140 \\
$2 \mathrm{~s}$ & 3 & 2 & 2 & 13.971 & 0.131 \\
$2 \mathrm{p}$ & 3 & 2 & 4 & 15.321 & 0.119 \\
$2 \mathrm{p}$ & 3 & 2 & 2 & 15.384 & 0.112 \\
$2 \mathrm{p}$ & 5 & 2 & 4 & 11.495 & 0.105 \\
$2 \mathrm{p}$ & 4 & 2 & 2 & 12.485 & 0.104 \\
\hline
\end{tabular}

Notes. Column $n_{i} l_{i}$ denotes the initial shell, $n_{j}$ denotes the final shell for optical electron transition, $g_{i}$ and $g_{j}$ denote the statistical weights for the initial and final levels, $\lambda$ denotes the line wavelength (in $\AA$ ), and $f_{i j}$ denote the absorption oscillator strength.

(This table is available in its entirety in a machine-readable form in the online journal. A portion is shown here for guidance regarding its form and content.)

\subsubsection{Transition Data}

We employ multiconfiguration wave functions determined after the Hamiltonian matrix diagonalization to calculate transition wavelengths $\lambda$, oscillator strengths $f$, and transition rates $A$ for excitation from the inner $n=2$ shell to the outer $n=3,4$, and 5 shells. Calculated line data cover the wavelength region $\lambda=12.606-14.096 \AA$ for $2 s-3 l$ transitions (a total of 17 lines), $\lambda=10.145-11.221 \AA$ for $2 s-4 l$ transitions (46 lines), and $\lambda=9.545-10.333 \AA$ for $2 s-5 l$ transitions (46 lines). Considering transitions from the $2 p$ shell, the wavelength range was $\lambda=13.594-17.246 \AA$ for the excitation of the $n=3$ shell ( 45 lines), $\lambda=11.316-12.934 \AA$ for the $n=4$ shell (111 lines), and $\lambda=10.525-11.689 \AA$ for the $n=5$ shell (111 lines).

In Table 1, we present data for Fe XVI transitions from the $2 s$ and $2 p$ shells to $n=3,4$, and 5 . We include only transitions with $f \geqslant 0.1$, whereas the more complete online version of the table contains lines having $f \geqslant 0.0001$. Weaker lines are not presented since they do not affect the ionization balance nor the final spectra. More details on data trimming are given in Section 3.2.

In Figure 1, we compare the strongest lines from our calculation with data derived using an MBPT (Gu et al. 2006), and with the Behar et al. (2001) results obtained using the multiconfiguration relativistic HULLAC computer package. The Behar et al. (2001) data are given for an $f$-value averaged wavelength $\lambda_{\text {av }}$ and the total oscillator strength $\sum f_{i j}$ of the line group, and therefore there is a sizable difference in the number of lines and in the values of $f$. Note that the point at $\lambda=17.296 \AA$ represents transitions from the $2 p$ to $3 s$ shells which are not considered in our calculations. When comparing our results with those of $\mathrm{Gu}$ et al. (2006), one can see quite good agreement both for wavelengths and oscillator strengths. There is some systematic shift toward shorter wavelengths in our data, as discussed in Kisielius et al. (2003). In addition, a significant difference with $\mathrm{Gu}$ et al. (2006) is the appearance in our results of a group of lines at $\lambda=11.5-12.5 \AA$ which represent transitions to $n=4$

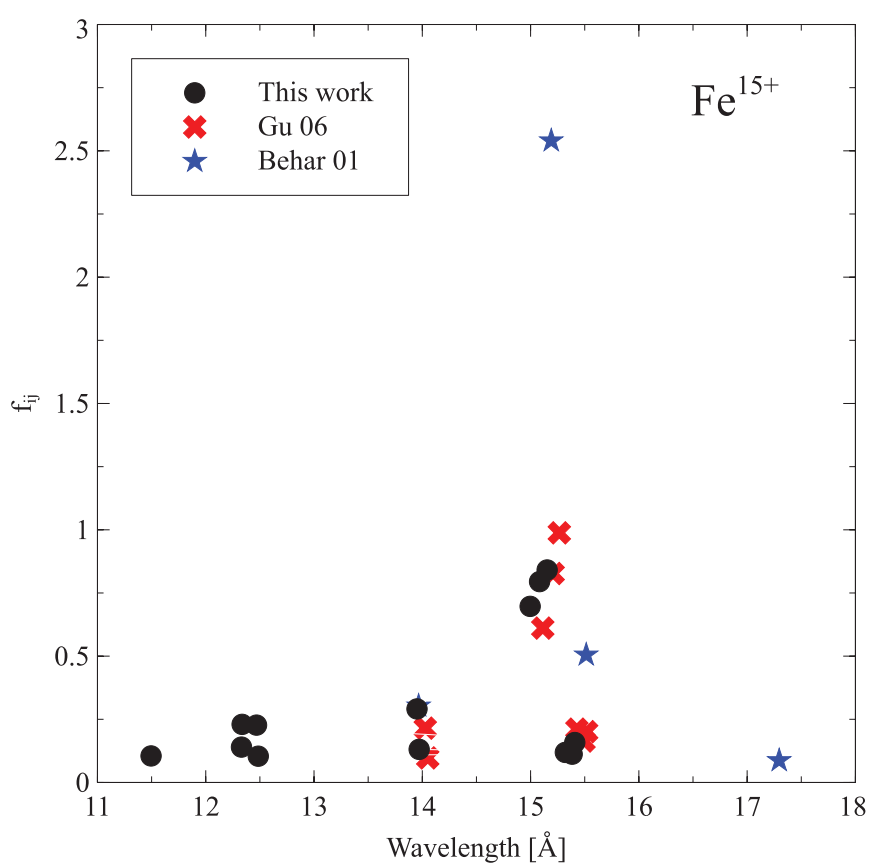

Figure 1. Comparison of the wavelengths $\lambda$ and the absorption oscillator strengths $f_{i j}$ for the strongest lines in Fe XVI. Dots represent our data, crosses are the results of Gu et al. (2006), and stars are those from Behar et al. (2001). The data at $\lambda=15 \AA$ represent $2 p-3 d$ UTA, at $\lambda=14 \AA$ represent $2 s-3 p$ UTA, at $\lambda=12.5 \AA$ represent $2 p-4 d$ UTA, and at $\lambda=11.5 \AA$ represent $2 p-5 d$ UTA. The Behar et al. (2001) data are given for an $f$-value averaged wavelength $\lambda_{\mathrm{av}}$ and the total oscillator strength $\sum f_{i j}$ of the line group.

(A color version of this figure is available in the online journal.)

and $n=5$ states. Some of these have large $f$-values and are important to include in atomic models.

The primary goal of the calculations presented here is to create an extensive set of inner-shell transition data to ensure that the total excitation and ionization rate is computed as accurately as possible. In a later section, we show how this new data change the ionization distribution of gas near an AGN. The line wavelengths we quote, which come from our atomic structure calculation, are no better or worse than the wavelengths quoted in previous studies. Indeed, the scatter in the wavelengths represents the uncertainty. Extensive sets of laboratory measurements of energy levels would be needed to improve the level energies and resulting wavelengths. Although a few experiments have been done (Brown et al. 2001; Simon et al. 2010), the extensive laboratory data needed to significantly improve the line wavelengths do not now exist.

\subsection{Fe Xv Lines}

The ground level of the $\mathrm{Fe}^{14+}$ ion is $1 s^{2} 2 s^{2} 2 p^{6} 3 s^{21} S_{0}^{\mathrm{e}}$. Its CI wave function expansion was chosen to include no more than two electrons virtually excited from the $2 p$ and $3 s$ shells in the case of the lines arising from the $2 p$ shell, and no more than two electrons excited from the $2 s$ and $3 s$ shells for the lines arising from the $2 s$ shell. We adopt the same configuration set both for the $2 p-3 l$ and the $2 p-4 l$ line calculations, but different radial orbital sets are used. For the $n=3$ line calculation, we employ the correlation radial orbitals $\overline{4} s, \overline{4} p, \overline{4} d$, and $\overline{4} f$, whereas the corresponding radial orbitals represent real states in the $n=4$ line calculations. This method contributes to an increased accuracy of the calculated wavelengths for $n=3$ lines which are much stronger than the $n=4$ ones. 


\subsubsection{Excitation from the $2 p$ Shell}

For excitation from $2 p$ to the $n=3$ and 4 shells, a CI wave function expansion was made of the $2 p^{6} 3 l^{2}(l=0,1,2)$, $2 p^{6} 3 s 3 d, 2 p^{5} 3 s^{2} 3 p, 2 p^{5} 3 p^{3}, 2 p^{5} 3 p 3 d^{2}$, and $2 p^{5} 3 s 3 p 3 d$ configurations containing two or three electrons within the $n=3$ shell. The even-parity configurations $2 p^{6} 3 l 4 l^{\prime}\left(l=0,1,2 ; l^{\prime}=\right.$ $0,1,2,3), 2 p^{5} 3 l^{2} 4 l^{\prime},\left(l=0,1,2 ; l^{\prime}=1,3\right), 2 p^{5} 3 l 3 l^{\prime} 4 l^{\prime \prime}(l=$ 0,$\left.1 ; l^{\prime}=0,1,2 ; l^{\prime \prime}=0,1,2,3\right)$ with a single electron virtually excited to the $n=4$ shell were added to that set as well as even-parity configurations $2 p^{6} 4 l^{2}(l=0,1,2,3)$, $2 p^{6} 4 s 4 d, 2 p^{6} 4 p 4 f, 2 p^{5} 3 p 4 l^{2}(l=0,1,2,3), 2 p^{5} 3 l 4 l^{\prime} 4 l^{\prime \prime}$ $\left(l=0,1,2 ; l^{\prime}=0,1,2 ; l^{\prime \prime}=1,2,3\right)$ with two electrons excited to the $n=4$ shell. In the case of inner $2 p$ shell excitation, we consider the ${ }^{1} S^{\mathrm{e}},{ }^{3} P^{\mathrm{e}}$, and ${ }^{5} D^{\mathrm{e}}$ terms which produce the $J=0$ fine-structure levels. Construction of such a basis produces 408 configuration state functions (CSFs) in the CI wave function expansion for the lower state of the $\mathrm{Fe}^{14+}$ ion.

Due to the electric dipole transition selection rules, we have to consider fine-structure levels with $J=1$ in the upper (odd) state of this ion. In the same way as for the lower (even) state, we construct three subsets of configurations in wave function expansion for the upper (odd) state. The CI wave function expansion consists of the $2 p^{6} 3 s 3 p, 2 p^{6} 3 p 3 d$, $2 p^{5} 3 s^{2} 3 d, 2 p^{5} 3 s 3 p^{2}, 2 p^{5} 3 s 3 d^{2}, 2 p^{5} 3 p^{2} 3 d$, and $2 p^{5} 3 d^{3}$ oddparity configurations which have two or three electrons in the $n=3$ shells. The odd-parity configurations $2 p^{6} 3 l 4 l^{\prime}(l=$ $\left.0,1,2 ; l^{\prime}=0,1,2,3\right), 2 p^{5} 3 l^{2} 4 l^{\prime}\left(l=0,1,2 ; l^{\prime}=0,2\right)$, and $2 p^{5} 3 l 3 l^{\prime} 4 l^{\prime \prime}\left(l=0,1 ; l^{\prime}=0,1,2 ; l^{\prime \prime}=0,1,2,3\right)$ with all possible electron distributions having a single electron virtually excited to the $n=4$ shell and the same parity configurations with two electrons in open $n=4$ shells $2 p^{6} 4 l 4 l^{\prime}\left(l=0,1,2 ; l^{\prime}=\right.$ $1,2,3), 2 p^{5} 3 l 4 l^{\prime 2}\left(l=0,2 ; l^{\prime}=0,1,2,3\right)$, and $2 p^{5} 3 l 4 l^{\prime} 4 l^{\prime \prime}$ $\left(l=0,1,2 ; l^{\prime}=0,1,2 ; l^{\prime \prime}=1,2,3\right)$ were added to that expansion.

In this construction of the CI wave function basis, we restricted our set by allowing no more than two electrons in the excited $n=4$ shell. Here, we consider the ${ }^{3} S^{\mathrm{o}},{ }^{1} P^{\mathrm{o}},{ }^{3} P^{\mathrm{o}}$, ${ }^{5} P^{\mathrm{o}},{ }^{3} D^{\mathrm{o}},{ }^{5} D^{\mathrm{o}}$, and ${ }^{5} F^{\mathrm{o}}$ terms which can produce fine-structure levels with $J=1$. As a result, we have the CI wave function expansion with 1094 CSFs for the upper states of the $\mathrm{Fe}^{14+}$ ion.

A very similar configuration set was applied when considering $2 p-5 l$ transitions. In this instance, the configurations with $n=4$ electrons were replaced by configurations with $n=5$. The only significant difference was the addition of the $2 p^{6} 3 l 4 l^{\prime}\left(l=0,1,2 ; l^{\prime}=0,1,2,3\right)$ even-parity configurations for in CI expansion of the lower state of $\mathrm{Fe}^{14+}$, and the $2 p^{6} 3 l 4 l^{\prime}$ $\left(l=0,1,2 ; l^{\prime}=0,1,2,3\right)$ odd-parity configurations for the excited state. Configurations with $5 g$ electrons were not included in the CI wave function expansion because transitions to such configurations are very weak. The total number of fine-structure levels with $J=0$ in this case was 413 for the lower configuration and 1107 for the upper configuration levels with $J=1$.

\subsubsection{Excitation from the 2 s Shell}

While determining the transition array $2 s-3 l, 4 l$ lines, for the CI expansion we have adopted the same subset (as in $2 p-3 l, 4 l$ case) of configurations with closed $2 s^{2}$ and $2 p^{6}$ electron shells and complemented it with configurations having one electron virtually excited from the $2 s$ shell. Specifically for the ground level, the even-parity configurations $2 s 2 p^{6} 3 s^{2} 3 d, 2 s 2 p^{6} 3 p^{2} 3 d, 2 s 2 p^{6} 3 s 3 p^{2}, 2 s 2 p^{6} 3 s 3 d^{2}$, $2 s 2 p^{6} 3 d^{3}, 2 s 2 p^{6} 3 l^{2} 4 l^{\prime}\left(l=0,1,2 ; l^{\prime}=0,2\right), 2 s 2 p^{6} 3 l 3 l^{\prime} 4 l^{\prime \prime}$ $\left(l=0,1 ; l^{\prime}=1,2 ; l^{\prime \prime}=0,1,2,3\right), 2 s 2 p^{6} 3 l 4 l^{\prime 2}\left(l=0,2 ; l^{\prime}=\right.$ $0,1,2,3)$, and $2 s 2 p^{6} 3 l 4 l^{\prime} 4 l^{\prime \prime}\left(l, l^{\prime}=0,1,2 ; l^{\prime \prime}=1,2,3\right)$, were included in our CI wave function expansion. These configurations were coupled in the ${ }^{1} S^{\mathrm{e}},{ }^{3} P^{\mathrm{e}}$, and ${ }^{5} D^{\mathrm{e}}$ non-relativistic $L S$-terms and produced 165 fine-structure levels with total orbital quantum number $J=0$.

The construction of the configuration set for the upper state is based on the same principles. We employ configurations with closed $2 s^{2}$ and $2 p^{6}$ electron shells from the $2 p-3 l, 4 l$ array calculation and add the configurations with an open $2 s$ shell. Following this method, we include the configurations $2 s 2 p^{6} 3 s^{2} 3 p, 2 s 2 p^{6} 3 p 3 d^{2}, 2 s 2 p^{6} 3 p^{3}$, and $2 s 2 p^{6} 3 s 3 p 3 d$ with the $2 s$ electron virtually excited to the $n=3$ shell, the oddparity configurations $2 s 2 p^{6} 3 l^{2} 4 l^{\prime}\left(l=0,1,2 ; l^{\prime}=1,3\right)$ and $2 s 2 p^{6} 3 l 3 l^{\prime} 4 l^{\prime \prime}\left(l=0,1,2 ; l^{\prime}=1,2 ; l^{\prime \prime}=0,1,2,3\right)$ with the $2 s$ electron virtually excited to the $n=4$ shell, and the odd-parity configurations with two electrons in the $n=4$ shell $2 s 2 p^{6} 3 l 4 l^{\prime} 4 l^{\prime \prime},\left(l=0,1,2 ; l^{\prime}=0,1,2 ; l^{\prime \prime}=1,2,3\right)$. Coupled to the ${ }^{3} S^{\mathrm{o}},{ }^{1} P^{\mathrm{o}},{ }^{3} P^{\mathrm{o}},{ }^{5} P^{\mathrm{o}},{ }^{3} D^{\mathrm{o}},{ }^{5} D^{\mathrm{o}}$, and ${ }^{5} F^{\mathrm{o}}$ terms, these configurations generate total 417 fine-structure levels with $J=1$.

For the transition array $2 s-5 l$, the wave function CI expansion was similar to that for the $2 s-4 l$ lines with the $n=4$ shell electrons replaced by $n=5$. The only addition was even-parity configurations $2 p^{6} 3 l 4 l^{\prime}\left(l=0,1,2 ; l^{\prime}=0,1,2,3\right)$ with one electron in the $4 l$ shell, included in the lower-state CI wave function expansion set, and the odd-parity configurations $2 p^{6} 3 l 4 l^{\prime}\left(l=0,1,2 ; l^{\prime}=0,1,2,3\right)$ in the upper-state wave function expansion. This CI expansion selection produces 170 fine-structure levels with $J=0$ for the ground state and 430 levels with $J=1$ for the excited state.

\subsubsection{Transition Data}

Similarly to the Fe XVI case, we obtained multiconfiguration wave functions to produce photoexcitation data for the Fe XV UTA lines. The calculated line data cover the wavelength region $\lambda=11.592-14.199 \AA$ for $2 s-3 l$ transitions (a total of 35 lines), $\lambda=9.620-11.423 \AA$ for $2 s-4 l$ transitions (147 lines), and $\lambda=9.545-10.333 \AA$ for $2 s-5 l$ transitions (147 lines). Considering transitions from the $2 p$ shell, the wavelength range was $\lambda=12.475-16.266 \AA$ for the excitation of the $n=3$ shell (88 lines), $\lambda=10.630-13.244 \AA$ for the $n=4$ shell ( 384 lines), and $\lambda=9.998-12.017 \AA$ for the $n=5$ shell (384 lines).

In Table 2, we present a sample of the line data for Fe XV. Transition wavelengths $\lambda$ and oscillator strengths $f_{i j}$ for excitation from the $2 s$ and $2 p$ shells to the outer $n=3,4$, and 5 shells are presented. Only a small portion of lines with $f \geqslant 0.04$ is included, with the more complete online version of the table containing all lines which have $f \geqslant 0.0001$. It is evident from Table 2 that the lines representing transitions to the $n=3$ and 4 shells are sufficiently strong as to affect spectral formation, and therefore they cannot be excluded from UTA line data sets.

Figure 2 compares the strongest lines from our calculations with the results derived using the MBPT approach $(\mathrm{Gu}$ et al. 2006) and with the relativistic data from Behar et al. (2001). In general, the agreement between all three sets of data is satisfactory. There is a systematic shift toward shorter wavelengths in our data set as noted for the Fe XVI lines.

\subsection{Fe XIV Lines}

The ground configuration of the $\mathrm{Fe}^{13+}$ ion is $1 s^{2} 2 s^{2} 2 p^{6} 3 s^{2} 3 p$. It has two fine-structure levels, ${ }^{2} P_{1 / 2}^{\mathrm{o}}$ and ${ }^{2} P_{3 / 2}^{\mathrm{o}}$, separated by 


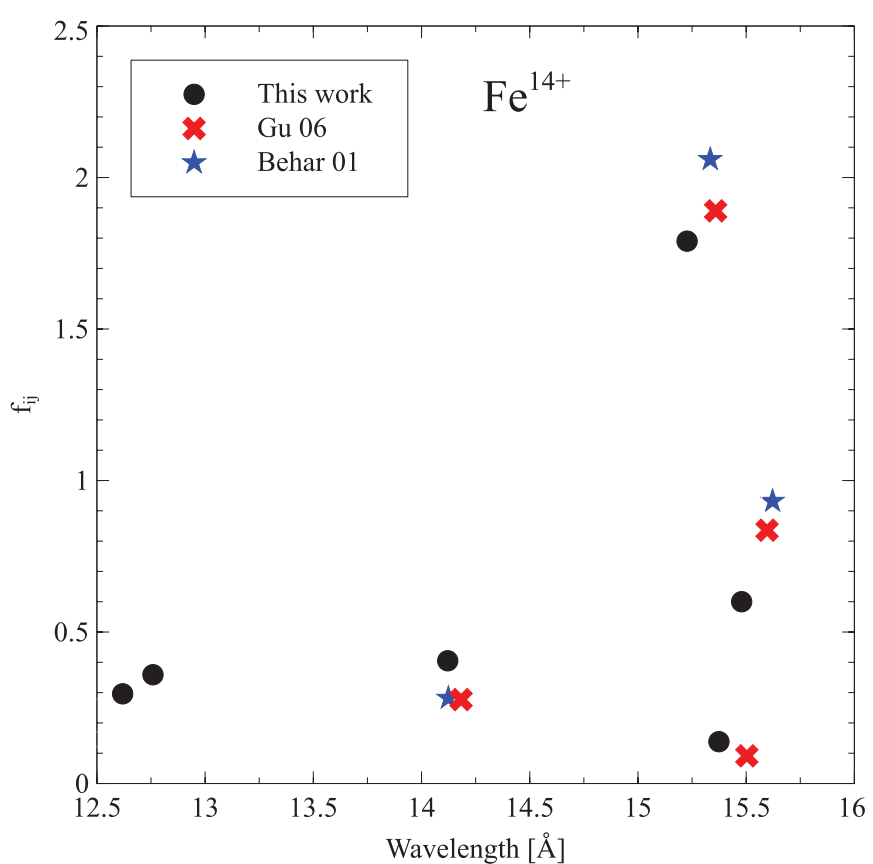

Figure 2. Comparison of the wavelengths $\lambda$ and absorption oscillator strengths $f_{i j}$ for the strongest lines in Fe XV. Dots represent our data, crosses are the results of Gu et al. (2006), and stars are those from Behar et al. (2001). The data at $\lambda=15.5 \AA$ represent $2 p-3 d$ UTA, those at $\lambda=14.1 \AA$ represent $2 s-3 p$ UTA, and those at $\lambda=12.7 \AA$ represent $2 p-4 d$ UTA. The Behar et al. (2001) data are given for an $f$-value averaged wavelength $\lambda_{\text {av }}$ and the total oscillator strength $\sum f_{i j}$ of the line group.

(A color version of this figure is available in the online journal.)

Table 2

Transitions $2 p-n_{j} d$ and $2 s-n_{j} p$ from the L Shell to the $n_{j}=3,4$, and 5 Shells in $\mathrm{Fe}^{14+}$

\begin{tabular}{lccccc}
\hline \hline$n_{i} l_{i}$ & $n_{j}$ & $g_{i}$ & $g_{j}$ & $\lambda(\AA)$ & $f_{i j}$ \\
\hline $2 \mathrm{p}$ & 3 & 1 & 3 & 15.227 & 1.790 \\
$2 \mathrm{p}$ & 3 & 1 & 3 & 15.479 & 0.600 \\
$2 \mathrm{~s}$ & 3 & 1 & 3 & 14.121 & 0.405 \\
$2 \mathrm{p}$ & 4 & 1 & 3 & 12.759 & 0.359 \\
$2 \mathrm{p}$ & 4 & 1 & 3 & 12.619 & 0.296 \\
$2 \mathrm{p}$ & 5 & 1 & 3 & 11.820 & 0.177 \\
$2 \mathrm{p}$ & 3 & 1 & 3 & 15.374 & 0.138 \\
$2 \mathrm{p}$ & 5 & 1 & 3 & 11.696 & 0.117 \\
$2 \mathrm{~s}$ & 4 & 1 & 3 & 11.407 & 0.097 \\
$2 \mathrm{p}$ & 4 & 1 & 3 & 12.615 & 0.065 \\
$2 \mathrm{p}$ & 3 & 1 & 3 & 16.008 & 0.060 \\
$2 \mathrm{p}$ & 3 & 1 & 3 & 15.779 & 0.041 \\
$2 \mathrm{~s}$ & 3 & 1 & 3 & 14.190 & 0.040 \\
\hline
\end{tabular}

Notes. Column $n_{i} l_{i}$ denotes the initial shell, $n_{j}$ denotes the final shell for optical electron transition, $g_{i}$ and $g_{j}$ denote the statistical weights for initial and final levels, $\lambda$ denotes the line wavelength (in $\AA$ ), and $f_{i j}$ denote the absorption oscillator strength.

(This table is available in its entirety in a machine-readable form in the online journal. A portion is shown here for guidance regarding its form and content.)

$2.33741 \mathrm{eV}$. Consequently, while considering electric-dipole transitions, we must determine energy levels with total angular momenta $J=1 / 2,3 / 2,5 / 2$ of the excited configuration of the $\mathrm{Fe}^{13+}$ ion.

We apply real radial orbitals for $1 s, 2 s, 2 p, 3 s, 3 p$, and $3 d$ electrons and correlation ones for the virtually excited $4 s, 4 p$, $4 d$, and $4 f$ electrons. This is consistent with the methods used to determine transition data for Fe XVI and Fe XV (see Kisielius et al. 2003), for photoexcitation from the $2 p$ and $2 s$ shells of the ground configuration to the levels of the excited $n=3$ configurations. Furthermore, for the excitation of the $n=4$ and $n=5$ lines, we adopt only real radial orbitals to describe $4 l$ and $5 l(l=0,1,2,3)$ electrons.

\subsubsection{Excitation from the $2 p$ Shell}

We employ the same configuration set both for calculations of $2 p-3 l$ and $2 p-4 l$ transition data. The CI expansion for the lower state includes configurations which have one or two electrons virtually excited from the $n=3$ shell. These are complemented with configurations which have the $2 p$ electron moved to the $n=3$ shell. The CI wave function expansion for the ground state was composed of the configurations with three or four electrons in $n=3$ shell $2 p^{6} 3 s^{2} 3 p, 2 p^{6} 3 p 3 d^{2}, 2 p^{6} 3 p^{3}, 2 p^{6} 3 s 3 p 3 d, 2 p^{5} 3 s^{2} 3 p^{2}$, $2 p^{5} 3 p^{2} 3 d^{2}, 2 p^{5} 3 s 3 d^{3}, 2 p^{5} 3 p^{4}, 2 p^{5} 3 d^{4}$, and $2 p^{5} 3 s 3 p^{2} 3 d$, the odd-parity configurations with one electron in virtually excited $n=4$ shell $2 p^{6} 3 l^{2} 4 l^{\prime}\left(l=0,1,2 ; l^{\prime}=1,3\right)$ and $2 p^{6} 3 l 3 l^{\prime} 4 l^{\prime \prime}$ $\left(l=0,1 ; l^{\prime}=1,2 ; l^{\prime \prime}=0,1,2,3\right)$, and the odd-parity configurations with two electrons in $n=4$ shell $2 p^{6} 3 l 4 l^{\prime} 4 l^{\prime \prime}(l=$ $\left.0,1,2 ; l^{\prime}=0,1,2 ; l^{\prime \prime}=1,2,3\right)$ and $2 p^{6} 3 p 4 l^{2}(l=0,1,2,3)$. In the case of the $J=1 / 2$ levels, we consider the ${ }^{2} S,{ }^{2} P,{ }^{4} P$, ${ }^{4} D,{ }^{6} D$, and ${ }^{6} F$ odd-parity $L S$-terms making up $336 \mathrm{CSF}$ in the CI wave function expansion, while for the $J=3 / 2$ finestructure levels, the odd-parity $L S$-terms ${ }^{2} P,{ }^{2} D,{ }^{4} S,{ }^{4} P,{ }^{4} D$, ${ }^{4} F,{ }^{6} P,{ }^{6} D,{ }^{6} F$, and ${ }^{6} G$ produce $553 \mathrm{CSFs}$.

For the upper state levels, the CI wave function expansion for the $2 p-4 l$ lines calculation is constructed from evenparity configuration sets. The configurations with electrons in $n=3$ shell $2 p^{6} 3 s^{2} 3 d, 2 p^{6} 3 s 3 p^{2}, 2 p^{6} 3 s 3 d^{2}, 2 p^{6} 3 p^{2} 3 d$, and $2 p^{6} 3 d^{3}$ the even-parity configurations with one electron in $n=4$ shell $2 p^{6} 3 l^{2} 4 l^{\prime}\left(l=0,1,2 ; l^{\prime}=0,2\right)$ and $2 p^{6} 3 l 3 l^{\prime} 4 l^{\prime \prime}\left(l=0,1 ; l^{\prime}=1,2 ; l^{\prime \prime}=0,1,2,3\right)$, the evenparity configurations with two electrons virtually excited into the $n=4$ shell, namely, $2 p^{6} 3 l 4 l^{\prime 2}\left(l=0,2 ; l^{\prime}=0,1,2,3\right)$ and $2 p^{6} 3 l 4 l^{\prime} 4 l^{\prime \prime}\left(l=0,1,2 ; l^{\prime}=0,1,2 ; l^{\prime \prime}=1,2,3\right)$ and the even-party configurations with a vacancy in the inner $2 p$ shell: $2 p^{5} 3 s^{2} 3 p 3 d, 2 p^{5} 3 s 3 p 3 d^{2}, 2 p^{5} 3 s 3 p^{3}, 2 p^{5} 3 p 3 d^{3}$, $2 p^{5} 3 p^{3} 3 d, 2 p^{5} 3 s^{2} 3 l 4 l^{\prime}\left(l=1,2 ; l^{\prime}=0,1,2,3\right), 2 p^{5} 3 s 3 l^{\prime 2} 4 l^{\prime \prime}$ $\left(l=0,1 ; l^{\prime}=1,2 ; l^{\prime \prime}=0,1,2,3\right), 2 p^{5} 3 l^{3} 4 l^{\prime}\left(l=1,2 ; l^{\prime}=\right.$ $0,1,2,3), 2 p^{5} 3 l^{2} 4 l^{\prime} 4 l^{\prime \prime},\left(l=0,1,2 ; l^{\prime}=0,1,2 ; l^{\prime \prime}=1,2,3\right)$, $2 p^{5} 3 l 3 l^{\prime} 4 l^{\prime \prime 2}\left(l=0,1 ; l^{\prime}=1,2 ; l^{\prime \prime}=0,1,2,3\right)$, and $2 p^{5} 3 l 3 l^{\prime} 4 l^{\prime \prime} 4 l^{\prime \prime \prime}\left(l=0,1 ; l^{\prime}=1,2 ; l^{\prime \prime}=0,1,2 ; l^{\prime \prime \prime}=1,2,3\right)$.

These configurations are bound to the ${ }^{2} S,{ }^{2} P,{ }^{4} P,{ }^{4} D,{ }^{6} P$, ${ }^{6} D$, and ${ }^{6} F L S$-terms for $J=1 / 2$ and produce 4099 CSFs in the CI wave function expansion. The terms ${ }^{2} P,{ }^{2} D,{ }^{4} S,{ }^{4} P,{ }^{4} D$, ${ }^{4} F,{ }^{6} P,{ }^{6} D,{ }^{6} F$, and ${ }^{6} G$ generate a total of 7049 fine-structure levels for $J=3 / 2$. For the total angular momentum $J=5 / 2$, the above configurations are bound to the ${ }^{2} D,{ }^{2} F,{ }^{4} P,{ }^{4} D,{ }^{4} F$, ${ }^{4} G,{ }^{6} S,{ }^{6} P,{ }^{6} D,{ }^{6} F,{ }^{6} G$, and ${ }^{6} H$ non-relativistic terms and produce 8211 levels.

The CI expansion for the lower state in the case of $2 p-$ $5 l$ consists of configuration sets similar to those used in $2 p-3 l, 4 l$ lines calculation. However, the four configuration complexes with $4 l(l=0,1,2,3)$ electrons are replaced by the configurations with $5 l$ electrons. Furthermore, the CI wave function expansion is extended by an additional set of the oddparity configurations $2 p^{6} 3 l^{2} 4 l^{\prime}\left(l=0,1,2 ; l^{\prime}=1,3\right)$ and $2 p^{6} 3 l 3 l^{\prime} 4 l^{\prime \prime}\left(l=0,1 ; l^{\prime}=1,2 ; l^{\prime \prime}=0,1,2,3\right)$ with one electron in an outer $n=4$ shell. The same (as in case of transitions to $n=3,4$ ) non-relativistic $L S$-terms produce 359 
CSFs in the CI wave function expansion for $J=1 / 2$, and 591 CSFs for $J=3 / 2$.

Similarly, the configuration sets with $4 l$ electrons are replaced by the configuration sets with $5 l$ electrons for the upper states. This set is extended with the even-parity configurations having one electron in the $n=4$ shell: $2 p^{6} 3 l^{2} 4 l^{\prime}\left(l=0,1,2 ; l^{\prime}=0,2\right)$ and $2 p^{6} 3 l 3 l^{\prime} 4 l^{\prime \prime}\left(l=0,1 ; l^{\prime}=1,2 ; l^{\prime \prime}=0,1,2,3\right)$. As in the previous case, even-parity $L S$-terms produce 4326, 7421, and $8612 \mathrm{CSF}$ in the CI wave function expansion for total angular momentum $J=1 / 2,3 / 2$, and $5 / 2$, respectively.

\subsubsection{Excitation from the $2 s$ Shell}

For the lines representing transitions from the inner $2 s$ shell to the valence $n=3$ or $n=4$ shells, the CI wave function expansion configurations for the lower state consists of the same configuration complexes for both cases. The first are configurations with outer electrons in $n=3$ shell $2 p^{6} 3 s^{2} 3 p$, $2 p^{6} 3 p^{3}, 2 p^{6} 3 p 3 d^{2}$, and $2 p^{6} 3 s 3 p 3 d$ and those with $2 s$ vacancy $2 s 2 p^{6} 3 s^{2} 3 p 3 d, 2 s 2 p^{6} 3 s 3 p 3 d^{2}, 2 s 2 p^{6} 3 s 3 p^{3}, 2 s 2 p^{6} 3 p^{3} 3 d$, and $2 s 2 p^{6} 3 p 3 d^{3}$. These configurations are complemented with odd-parity configurations with one and two electrons in the $4 l$ shell: $2 p^{6} 3 l^{2} 4 l^{\prime}\left(l=0,1,2 ; l^{\prime}=1,3\right), 2 p^{6} 3 l 3 l^{\prime} 4 l^{\prime \prime}(l=$ 0,$\left.1 ; l^{\prime}=1,2 ; l^{\prime \prime}=0,1,2,3\right), 2 p^{6} 3 l 4 l^{\prime} 4 l^{\prime \prime}\left(l=0,1,2 ; l^{\prime}=\right.$ $\left.0,1,2 ; l^{\prime \prime}=1,2,3\right), 2 p^{6} 3 p 4 l^{2}(l=0,1,2,3), 2 s 2 p^{6} 3 l^{2} 3 l^{\prime} 4 l^{\prime \prime}$ $\left(l=0,1 ; l^{\prime}=1,2 ; l^{\prime \prime}=0,1,2,3\right), 2 s 2 p^{6} 3 l 3 l^{\prime 2} 4 l^{\prime \prime}(l=$ 0,$\left.1 ; l^{\prime}=1,2 ; l^{\prime \prime}=0,1,2,3\right), 2 s 2 p^{6} 3 l^{3} 4 l^{\prime}\left(l=1,2 ; l^{\prime}=\right.$ $0,1,2,3)$, and $2 s 2 p^{6} 3 s 3 p 3 d 4 l(l=0,2)$.

In the case of the $J=1 / 2$ levels, the above configurations are bound to the ${ }^{2} S,{ }^{2} P,{ }^{4} P,{ }^{4} D,{ }^{6} D$, and ${ }^{6} F$ odd-parity $L S$ terms which yield a total of 660 CSFs in the CI wave function expansion. For the $J=3 / 2$ levels represented by the ${ }^{2} P,{ }^{2} D$, ${ }^{4} S,{ }^{4} P,{ }^{4} D,{ }^{4} F,{ }^{6} P,{ }^{6} D,{ }^{6} F$, and ${ }^{6} G$ odd-parity $L S$-terms, the wave function expansion consists of 1102 CSFs.

The wave function expansion for the lower state in the case of the $2 s-5 l$ transition array is constructed in a similar way by replacing all the configurations containing $4 l(l=$ $0,1,2,3$ ) electrons with configurations containing $5 l$ electrons. Furthermore, a complex of configurations with one electron in the $4 l$ shell is added. This extends CI expansion by including odd-parity configurations $2 p^{6} 3 l^{2} 4 l^{\prime}\left(l=0,1,2 ; l^{\prime}=1,3\right)$ and $2 p^{6} 3 l 3 l^{\prime} 4 l^{\prime \prime}\left(l=0,1 ; l^{\prime}=1,2 ; l^{\prime \prime}=0,1,2,3\right)$. The same (as for the $n=4$ lines) $L S$-terms are considered, and the CI wave function expansion gives rise to 716 and 1193 CSFs for the $J=1 / 2$ and $J=3 / 2$ levels, respectively.

While considering the upper state of $\mathrm{Fe}^{13+}$, the CI wave function expansion for $2 s-3 l, 4 l$ lines is constructed of several configuration complexes, representing configurations with one or two electrons virtually excited from the ground configuration. We have included configurations $2 p^{6} 3 s^{2} 3 d, 2 p^{6} 3 s 3 p^{2}$, $2 p^{6} 3 s 3 d^{2}, 2 p^{6} 3 p^{2} 3 d$, and $2 p^{6} 3 d^{3}$ with three outer electrons in $n=3$ shell, the even-parity configurations $2 p^{6} 3 l^{2} 4 l^{\prime}$ $\left(l=0,1,2 ; l^{\prime}=0,2\right), 2 p^{6} 3 l 3 l^{\prime} 4 l^{\prime \prime}\left(l=0,1 ; l^{\prime}=1,2 ; l^{\prime \prime}=\right.$ $0,1,2,3)$, and $2 p^{6} 3 l 4 l^{\prime 2}\left(l=0,2 ; l^{\prime}=0,1,2,3\right)$ with one electron in outer $n=4$ shell, $2 p^{6} 3 l 4 l^{\prime} 4 l^{\prime \prime}\left(l=0,1,2 ; l^{\prime}=\right.$ $\left.0,1,2 ; l^{\prime \prime}=1,2,3\right)$ with two electrons in $n=4$ shell. On top of that, we have extended the CI expansion by adding the even-parity configurations with a vacancy in the $2 s$ shell: $2 s 2 p^{6} 3 l^{2} 3 l^{\prime 2}\left(l=0,1 ; l^{\prime}=1,2\right), 2 s 2 p^{6} 3 s 3 p^{2} 3 d, 2 s 2 p^{6} 3 s 3 d^{3}$, $2 s 2 p^{6} 3 p^{4}, 2 s 2 p^{6} 3 d^{4}, 2 s 2 p^{6} 3 l^{2} 3 l^{\prime} 4 l^{\prime \prime}\left(l=0,1 ; l^{\prime}=1,2 ; l^{\prime \prime}=\right.$ $0,1,2,3), 2 s 2 p^{6} 3 l 3 l^{\prime 2} 4 l^{\prime \prime}\left(l=0,1 ; l^{\prime}=1,2 ; l^{\prime \prime}=0,1,2,3\right)$, $2 s 2 p^{6} 3 l^{3} 4 l^{\prime}\left(l=1,2 ; l^{\prime}=0,1,2,3\right), 2 s 2 p^{6} 2 s 2 p 3 d 4 l(l=$ $1,3), 2 s 2 p^{6} 3 l^{2} 4 l^{\prime 2}\left(l=0,1,2 ; l^{\prime}=0,1,2,3\right), 2 s 2 p^{6} 3 l^{2} 4 l^{\prime} 4 l^{\prime \prime}$ $\left(l=0,1,2 ; l^{\prime}=0,1 ; l^{\prime \prime}=2,3\right), 2 s 2 p^{6} 3 s 3 d 4 l^{2}(l=$
Table 3

Transitions $2 p-n_{j} d$ and $2 s-n_{j} p$ from the L Shell to the $n_{j}=3,4$, and 5 Shells in $\mathrm{Fe}^{13+}$

\begin{tabular}{llllcc}
\hline \hline$n_{i} l_{i}$ & $n_{j}$ & $g_{i}$ & $g_{j}$ & $\lambda(\AA)$ & $f_{i j}$ \\
\hline $2 \mathrm{p}$ & 3 & 2 & 4 & 15.474 & 0.920 \\
$2 \mathrm{p}$ & 3 & 4 & 6 & 15.402 & 0.430 \\
$2 \mathrm{p}$ & 3 & 2 & 2 & 15.433 & 0.394 \\
$2 \mathrm{p}$ & 3 & 4 & 6 & 15.507 & 0.393 \\
$2 \mathrm{p}$ & 3 & 4 & 4 & 15.449 & 0.378 \\
$2 \mathrm{p}$ & 3 & 4 & 2 & 15.408 & 0.310 \\
$2 \mathrm{p}$ & 3 & 4 & 4 & 15.375 & 0.266 \\
$2 \mathrm{p}$ & 3 & 4 & 6 & 15.645 & 0.209 \\
$2 \mathrm{p}$ & 3 & 2 & 2 & 15.310 & 0.201 \\
$2 \mathrm{p}$ & 3 & 2 & 4 & 15.499 & 0.194 \\
$2 \mathrm{p}$ & 3 & 4 & 4 & 15.665 & 0.164 \\
$2 \mathrm{~s}$ & 3 & 2 & 4 & 14.282 & 0.157 \\
$2 \mathrm{~s}$ & 3 & 2 & 2 & 14.300 & 0.147 \\
$2 \mathrm{p}$ & 3 & 2 & 4 & 15.522 & 0.147 \\
$2 \mathrm{p}$ & 4 & 4 & 6 & 12.891 & 0.159 \\
$2 \mathrm{p}$ & 4 & 2 & 4 & 12.921 & 0.109 \\
$2 \mathrm{p}$ & 3 & 2 & 2 & 15.697 & 0.103 \\
\hline
\end{tabular}

Notes. Column $n_{i} l_{i}$ denotes the initial shell, $n_{j}$ denote the final shell for optical electron transition, $g_{i}$ and $g_{j}$ denote the statistical weights for the initial and final levels, $\lambda$ denote the line wavelength (in $\AA$ ), and $f_{i j}$ denote the absorption oscillator strength.

(This table is available in its entirety in a machine-readable form in the online journal. A portion is shown here for guidance regarding its form and content.)

$0,1,2,3)$, and $2 s 2 p^{6} 3 l 3 l^{\prime} 4 l^{\prime \prime} 4 l^{\prime \prime \prime}\left(l=0,1 ; l^{\prime}=1,2 ; l^{\prime \prime}=\right.$ $\left.0,1,2 ; l^{\prime \prime \prime}=1,2,3\right)$. We consider the same $L S$-terms as in the transitions from the $2 p$ shell. Consequently, the numbers of CSFs included in the CI wave function expansion are 1695,2865 , and 3270 for the $J=1 / 2,3 / 2$, and $5 / 2$ levels, respectively.

While constructing the upper-state CI wave function expansion configuration set for the $2 s-5 l$ transition array, we apply the same principles as in the $n=4$ case but replace the configuration sets containing $4 l$ electrons with those containing $5 l$ electrons. That expansion is extended with a set of evenparity configurations with a valence $n=4$ electron $2 p^{6} 3 l^{2} 4 l^{\prime}$ $\left(l=0,1,2 ; l^{\prime}=0,2\right)$ and $2 p^{6} 3 l 3 l^{\prime} 4 l^{\prime \prime}\left(l=0,1 ; l^{\prime}=1,2 ; l^{\prime \prime}=\right.$ $0,1,2,3)$. This set generates 1751,2954 , and 3364 CSFs in the CI wave function expansion for the $J=1 / 2,3 / 2$, and $5 / 2$ levels, respectively.

\subsubsection{Transition Data}

Using the above methods to generate the CI wave function expansions, we obtained wave functions to produce photoexcitation line data for the Fe XIV ion. Calculated line data cover the wavelength region $\lambda=10.571-14.432 \AA$ for $2 s-3 l$ transitions (a total of 617 lines), $\lambda=9.298-11.707 \AA$ for $2 s-4 l$ transitions (3194 lines), and $\lambda=8.890-10.861 \AA$ for $2 s-5 l$ transitions (147 lines). For transitions from the $2 p$ shell, the wavelength range was $\lambda=12.341-16.626 \AA$ for excitation from the $n=3$ shell (1343 lines), $\lambda=10.141-13.642 \AA$ for the $n=4$ shell (7425 lines), and $\lambda=9.685-12.438 \AA$ for the $n=5$ shell (8640 lines).

Table 3 lists the atomic data for the Fe XIV UTA lines. Transition wavelengths $\lambda$ and absorption oscillator strengths $f_{i j}$ for excitation from the $2 s$ and $2 p$ shells to the outer $n=3,4$, and 5 shells are given for lines with $f \geqslant 0.1$. A more extensive data set is presented in the online version of this 


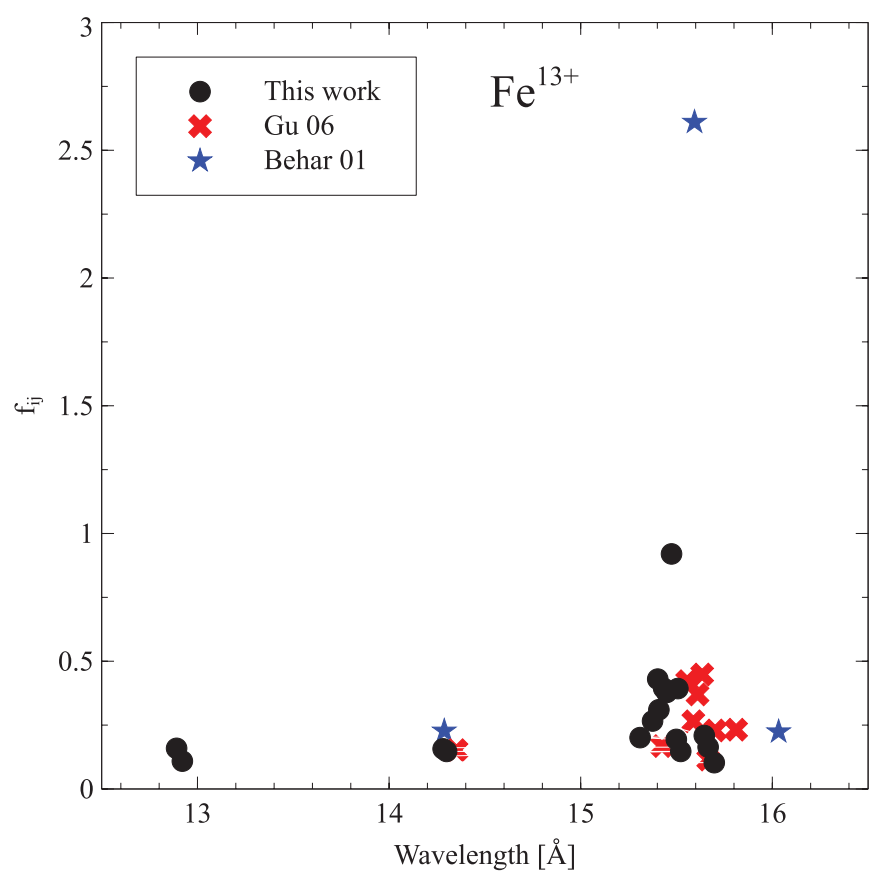

Figure 3. Comparison of the wavelengths $\lambda$ and absorption oscillator strengths $f_{i j}$ for the strongest lines in Fe XIV. Dots represent our data, crosses are the results of Gu et al. (2006), and stars are those from Behar et al. (2001). The data at $\lambda=15.5 \AA$ represent the $2 p-3 d$ UTA, those at $\lambda=14.3 \AA$ represent the $2 s-3 p$ UTA, and those at $\lambda=12.9 \AA$ represent the $2 p-4 d$ UTA. The Behar et al. (2001) data are given for an $f$-value averaged wavelength $\lambda_{\text {av }}$ and the total oscillator strength $\sum f_{i j}$ of the line group.

(A color version of this figure is available in the online journal.)

table, where we provide results for all lines with $f \geqslant 0.0001$ (see Section 3.2 for more details about data trimming).

In Figure 3, we compare the strongest lines from our calculation with the results derived using the MBPT approach of $\mathrm{Gu}$ et al. (2006) and with the HULLAC relativistic data from Behar et al. (2001). There is reasonable wavelength agreement for all three sets, as found for Fe XVI and Fe XV. The only exception is the line with $\lambda=16.033 \AA$ from Behar et al. (2001), with the larger values of oscillator strengths $\sum f_{i j}$ found by these authors explained in Section 2.1.3. A wavelength disagreement between our results and the MBPT data was also discussed in that section.

\section{INCORPORATION INTO CLOUDY}

\subsection{Data Sources}

The UTA data sets now used in Cloudy are summarized in Table 4. The Opacity Project (OP; Badnell et al. 2005) produced complete data, for the $\mathrm{K}$ shell as well as the L1 and L2 shells where appropriate, for ions with 12 or fewer bound electrons. These include the autoionization branching ratio which we consider in calculating the ionization rate. The OP data are marked "B" in this table. Gu et al. (2006) provide all stages of ionization for $\mathrm{Fe}$ but includes only excitations from the $2 p$ shell, denoted by " $G$ " in the table. These data sets have been included in Cloudy since shortly after their original publication dates.

The data presented in this paper are now being incorporated into Cloudy and are denoted by " $\mathrm{K}$ " in Table 4. Two ions, $\mathrm{Fe}^{14+}$ and $\mathrm{Fe}^{15+}$, are present in both the current data and the OP. We use the OP data for excitations from the $\mathrm{K}$ shell and the current results for $\mathrm{L}$ shell excitations. Our new data do not include autoionization rates, so these were copied from $\mathrm{Gu}$ et al. (2006)

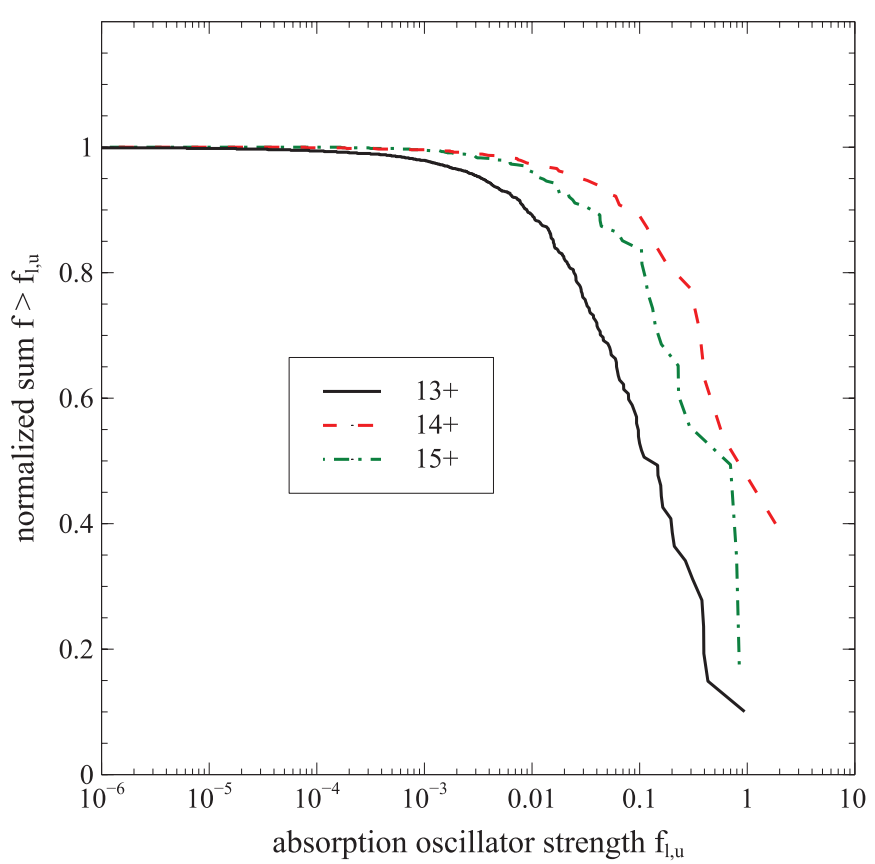

Figure 4. Absorption line oscillator strength $f_{l, u}$ plotted against the sum of all lines with $f>f_{l, u}$. The $y$-axis has been scaled by the sum of the oscillator strengths. The integrated absorption is well converged when only lines with $f>10^{-4}$ are included.

(A color version of this figure is available in the online journal.)

for the five most important lines of Fe XV and Fe XVI. For other lines, we assume that the branching ratio for ionization is $100 \%$. In the default setup of the code, we only use transitions from the ground level for all data sources. See also the discussion in Section 4.1.

It is clear from Table 4 that many ions simply have no current data. For example, there are no $\mathrm{K}$ shell data for $\mathrm{Fe}^{13+}$. These are all major requirements for accurate spectral simulations.

\subsection{Data Trimming}

The newly computed data sets are quite extensive and include a large number of exceptionally weak lines. Figure 4 shows the absorption line oscillator strength $f_{l, u}$ plotted against the normalized sum of all lines with $f>f_{l, u}$. Careful examination of such data shows that the summed oscillator strength, which affects the UTA ionization rate, has converged to within $0.57 \%$, $0.15 \%$, and $0.08 \%$ of the total for $\mathrm{Fe}^{13+}, \mathrm{Fe}^{14+}$, and $\mathrm{Fe}^{15+}$, when all lines with $f \geqslant 10^{-4}$ are included. Weaker lines have little effect upon the ionization rate because the line-center opacity is smaller than the continuous photoionization opacity. Accordingly, we only consider lines with $f \geqslant 10^{-4}$, although we retain all data for future flexibility. For the case of $\mathrm{Fe}^{13+}$, this reduces the number of lines from 80894 to 754 , for $\mathrm{Fe}^{14+}$ from 2738 to 103 , and for $\mathrm{Fe}^{15+}$ from 595 to 137.

\subsection{Damping Parameters and Line Broadening}

The Voigt function, which describes the line profile including both thermal and natural broadening, is given by

$$
H(a, x)=\frac{a}{\pi} \int_{-\infty}^{+\infty} \frac{\exp \left(-y^{2}\right)}{(x-y)^{2}+a^{2}} d y,
$$


Table 4

Data Sources used in the Default Setup of the Cloudy Code

\begin{tabular}{|c|c|c|c|c|c|c|c|c|c|c|c|c|c|c|c|c|c|c|c|c|c|c|c|c|c|c|c|c|c|c|c|}
\hline Ion & 0 & 1 & 2 & 3 & 4 & 5 & 6 & 7 & 8 & 9 & 10 & 11 & 12 & 13 & 14 & 15 & 16 & 17 & 18 & 19 & 20 & 21 & 22 & 23 & 24 & 25 & 26 & 27 & 28 & 29 & 30 \\
\hline \multicolumn{32}{|l|}{$\mathrm{H}$} \\
\hline $\mathrm{He}$ & & & & & & & & & & & & & & & & & & & & & & & & & & & & & & & \\
\hline $\mathrm{Li}$ & B & & & & & & & & & & & & & & & & & & & & & & & & & & & & & & \\
\hline $\mathrm{Be}$ & B & B & & & & & & & & & & & & & & & & & & & & & & & & & & & & & \\
\hline B & B & B & B & & & & & & & & & & & & & & & & & & & & & & & & & & & & \\
\hline C & B & B & B & B & & & & & & & & & & & & & & & & & & & & & & & & & & & \\
\hline $\mathrm{N}$ & B & B & B & B & B & & & & & & & & & & & & & & & & & & & & & & & & & & \\
\hline $\mathrm{O}$ & B & B & B & B & B & B & & & & & & & & & & & & & & & & & & & & & & & & & \\
\hline F & B & B & B & B & B & B & B & & & & & & & & & & & & & & & & & & & & & & & & \\
\hline $\mathrm{Ne}$ & B & B & B & B & B & B & B & B & & & & & & & & & & & & & & & & & & & & & & & \\
\hline $\mathrm{Na}$ & B & B & B & B & B & B & B & B & B & & & & & & & & & & & & & & & & & & & & & & \\
\hline $\mathrm{Mg}$ & B & B & B & B & B & B & B & B & B & B & & & & & & & & & & & & & & & & & & & & & \\
\hline $\mathrm{Al}$ & & B & B & B & B & B & B & B & B & B & B & & & & & & & & & & & & & & & & & & & & \\
\hline $\mathrm{Si}$ & & & B & B & B & B & B & B & B & B & B & B & & & & & & & & & & & & & & & & & & & \\
\hline $\mathrm{P}$ & & & & B & B & B & B & B & B & B & B & B & B & & & & & & & & & & & & & & & & & & \\
\hline$S$ & & & & & B & B & B & B & B & B & B & B & B & B & & & & & & & & & & & & & & & & & \\
\hline $\mathrm{Cl}$ & & & & & & B & B & B & B & B & B & B & B & B & B & & & & & & & & & & & & & & & & \\
\hline $\mathrm{Ar}$ & & & & & & & B & B & B & B & B & B & B & B & B & B & & & & & & & & & & & & & & & \\
\hline K & & & & & & & & B & B & B & B & B & B & B & B & B & B & & & & & & & & & & & & & & \\
\hline $\mathrm{Ca}$ & & & & & & & & & B & B & B & B & B & B & B & B & B & B & & & & & & & & & & & & & \\
\hline $\mathrm{Sc}$ & & & & & & & & & & B & B & B & B & B & B & B & B & B & B & & & & & & & & & & & & \\
\hline $\mathrm{Ti}$ & & & & & & & & & & & B & B & B & B & B & B & B & B & B & B & & & & & & & & & & & \\
\hline V & & & & & & & & & & & & B & B & B & B & B & B & B & B & B & B & & & & & & & & & & \\
\hline $\mathrm{Cr}$ & & & & & & & & & & & & & B & B & B & B & B & B & B & B & B & B & & & & & & & & & \\
\hline $\mathrm{Mn}$ & & & & & & & & & & & & & & B & B & B & B & B & B & B & B & B & B & & & & & & & & \\
\hline $\mathrm{Fe}$ & $\mathrm{G}$ & $\mathrm{G}$ & $\mathrm{G}$ & $\mathrm{G}$ & G & $\mathrm{G}$ & G & G & $\mathrm{G}$ & G & $\mathrm{G}$ & $\mathrm{G}$ & $\mathrm{G}$ & $\mathrm{K}$ & BK & $\mathrm{BK}$ & B & B & B & B & B & $\mathrm{B}$ & B & B & & & & & & & \\
\hline Co & & & & & & & & & & & & & & & & B & B & B & B & B & B & B & B & B & B & & & & & & \\
\hline $\mathrm{Ni}$ & & & & & & & & & & & & & & & & & B & B & B & B & B & B & B & B & B & B & & & & & \\
\hline $\mathrm{Cu}$ & & & & & & & & & & & & & & & & & & B & B & B & B & $\mathrm{B}$ & B & B & B & B & B & & & & \\
\hline $\mathrm{Zn}$ & & & & & & & & & & & & & & & & & & & B & B & B & B & B & B & B & B & B & B & & & \\
\hline Ion & 0 & 1 & 2 & 3 & 4 & 5 & 6 & 7 & 8 & 9 & 10 & 11 & 12 & 13 & 14 & 15 & 16 & 17 & 18 & 19 & 20 & 21 & 22 & 23 & 24 & 25 & 26 & 27 & 28 & 29 & 30 \\
\hline
\end{tabular}

Notes. B: Badnell et al. (2005) data, G: Gu et al. (2006) data, and K: present results.

where $x$ is the displacement from line center, measured in terms of the Doppler width $\Delta v_{\text {Dop }}$,

$$
x \equiv \frac{v-v_{0}}{\Delta v_{\text {Dop }}} .
$$

The damping parameter $a$ is the ratio of natural (radiation damped) to thermal line widths

$$
a \equiv \frac{\gamma}{4 \pi \Delta v_{\text {Dop }}}
$$

and the natural broadening width is given by the damping constant $\gamma$ which is expressed as a sum of radiative $A^{r}$ and autoionization $A^{a}$ rates

$$
\gamma=\sum_{l<u} A_{u l}^{r}+\sum_{l^{\prime}<u} A_{u l^{\prime}}^{a}
$$

$H(a, x)$ is normalized so that its integral over $x$ is $\sqrt{\pi}$.

The Voigt function must be evaluated to account for line selfshielding when finite column densities are encountered, while the total radiative decay ratio out of the upper level is needed to derive the damping constant $\gamma$. This lifetime is the sum of the autoionization and radiative decay rates. Our new data do not include calculations of autoionization rates (except for the five lines mentioned earlier). However, in order to calculate the damping constant $\gamma$ we need to assume a value, so we adopted the value, $10^{15} \mathrm{~s}^{-1}$, taken from OP results for Fe XV and Fe XVI. The other data sources include autoionization rates, which we adopt.

Figure 5 shows the resulting damping parameters, plotted as a function of transition wavelength, for a gas kinetic temperature of $10^{4} \mathrm{~K}$. UTA transitions are strongly damped, often with $a \gg 1$, because of the rapid autoionization rate. For comparison, the damping parameter for a strong UV line, such as $\mathrm{H}_{\mathrm{I}} \mathrm{L} \alpha$, is $a \sim 4 \times 10^{-4}$. It should be noted that the high damping parameter $a$ is a consequence of the inner-shell nature of these excitations regardless of their being in UTAs.

While simple approximations to the Voigt function may be sufficient for UV-optical spectroscopy, with small $a$, the large range in damping parameter that occurs when UTAs are considered make it important to use implementations of the Voigt function which are accurate for all $a$. We adopt the routine provided by Wells (1999), combined with a specially designed faster routine for $a \leqslant 0.1$, which provides results with a relative accuracy of 1 in $10^{4}$, and has been confirmed to pass all the test cases given by Zaghloul \& Ali (2011).

Stellar atmosphere texts (Rutten 2003; Mihalas 1978) often focus on results derived for approximations to the Voigt function which, while valid in the original context, are not accurate for the full range of frequency and $a$ needed to include UTA transitions. Figure 6 shows the function for a typical strong UV line such as H I Ly $\alpha\left(a \sim 10^{-4}\right)$ and a strongly damped UTA transition $\left(a \sim 10^{3}\right)$. We see that at line center $H(a, x) \sim(1+a)^{-1}$, and that the core of the line is roughly $x \sim(1+a)$ wide. 


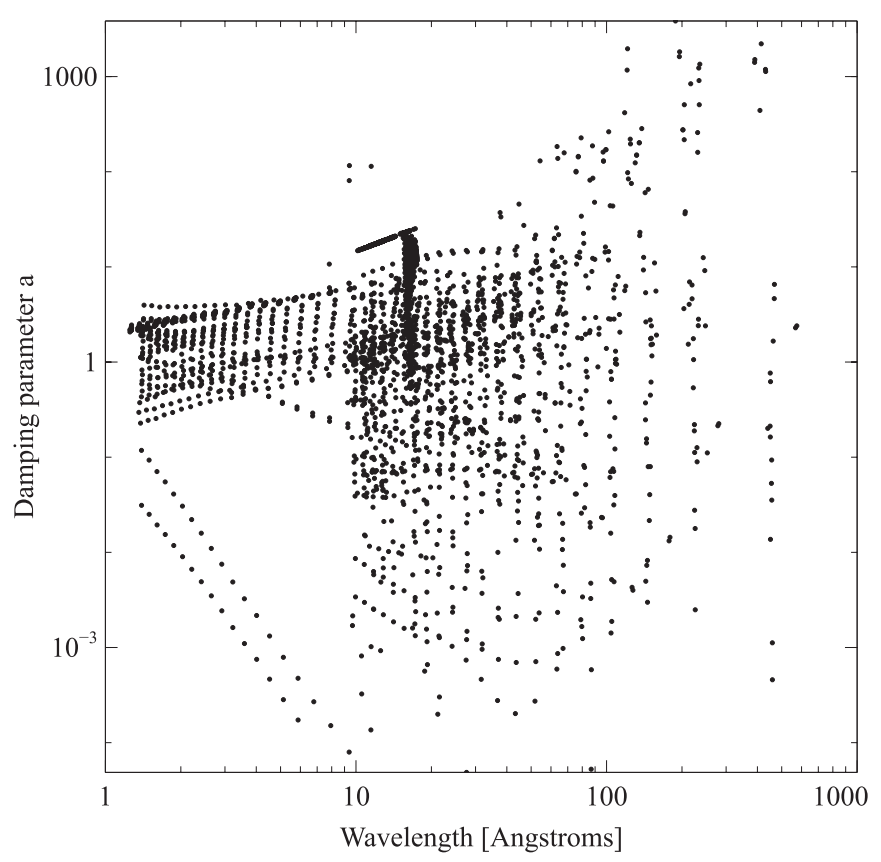

Figure 5. Damping parameters as a function of the line wavelength for our complete set of UTA lines.

There are a large number of lines which strongly overlap to produce the observed UTA features. The effects of mutual line shielding due to overlap must be included if the ionization rate is to be properly computed. Line overlap is treated using a combination of a coarse, low-resolution continuum, and a fine continuum, with resolution sufficient to resolve lines, as described in Shaw et al. (2005).

\section{APPLICATION TO AGN}

\subsection{Fe XIV as a Density Indicator}

Our Fe XIV data include both fine-structure levels within the ground term, namely, ${ }^{2} P_{1 / 2}^{\mathrm{o}}$ and ${ }^{2} P_{3 / 2}^{\mathrm{o}}$. These are separated by $2.33741 \mathrm{eV}$ and produce the famous "green" Fe XIV coronal line at $5303 \AA$. There are approximately the same number of UTA lines originating from either level in the overall configuration.

The critical density of the excited $J=3 / 2$ level is $\sim 3 \times$ $10^{9} \mathrm{~cm}^{-3}$ at $10^{5} \mathrm{~K}$. The temperature $T=10^{5} \mathrm{~K}$ is approximately that of the formation of the Fe ions discussed in current paper. At considerably lower densities most of the population will be in the $J=1 / 2$ level, while for high temperatures and densities the levels will be populated according to their statistical weight and most will be in the excited level. If the absorption characteristics are different in these two different limits then the UTA lines could be used as a density indicator.

Figure 7 (right frame) shows how the profile of the $\lambda 15.5$ Fe XIV UTA changes with density. The calculation assumed a total hydrogen column density of $10^{21} \mathrm{~cm}^{-2}$, a solar Fe abundance, and two hydrogen densities, $n_{\mathrm{H}}=10^{5} \mathrm{~cm}^{-3}$, sufficiently low for all populations to lie in the lower $J=1 / 2$ level, and $n_{\mathrm{H}}=10^{15} \mathrm{~cm}^{-3}$, high enough for the levels to be populated according to their statistical weights. Significant differences are present. The features originating from the configurations which include the excited $J=3 / 2$ level are not present in the low-density profile. At the high-density limit, the ratio of the excited and lower level population is $2: 1$.

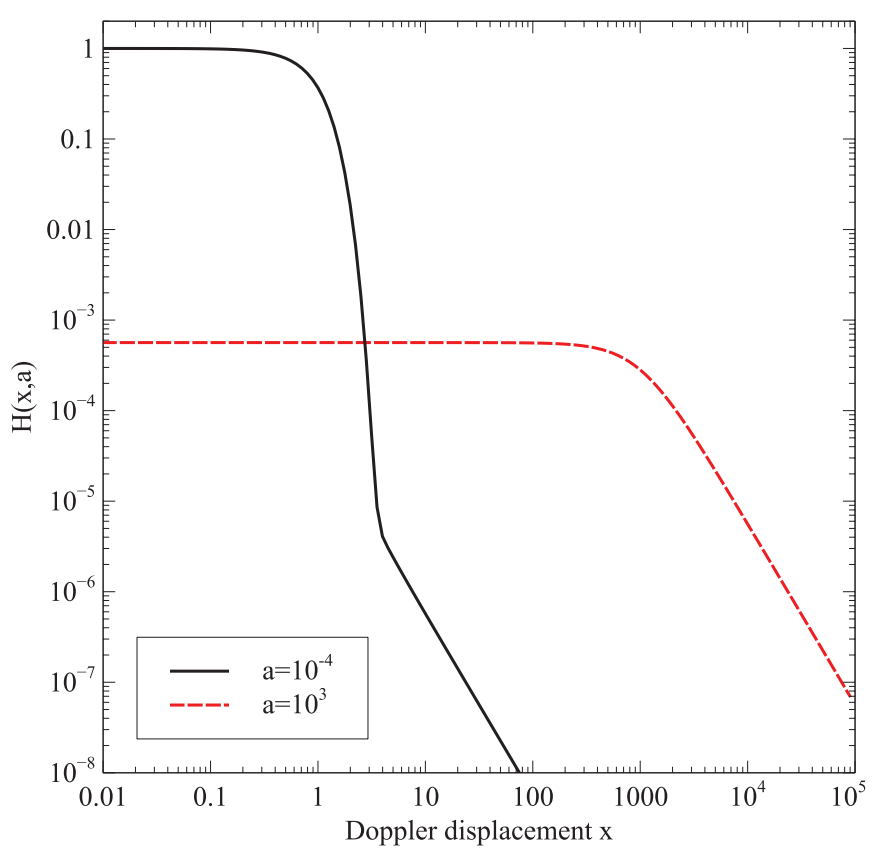

Figure 6. Voigt function $H(a, x)$ for two values of the damping parameter $a$. (A color version of this figure is available in the online journal.)

Therefore, new features connected to the $J=3 / 2$ level (e.g., $\lambda 15.35, \lambda 15.40$, and $\lambda 15.65)$ become evident, whereas features originating from the ground $J=1 / 2$ level (e.g., $\lambda 15.30, \lambda 15.43$, and $\lambda 15.47$ ) become less pronounced. The total absorption does not change because the sum of the absorption oscillator strengths in this wavelength region is approximately the same both for $J=3 / 2$ and $J=1 / 2$ levels, although different transitions are produced by each level.

Figure 7 (left frame) shows the effects of density in the area of the $\lambda 13.0$ UTA lines in Fe XIV. It demonstrates very similar behavior to the case of the $\lambda 15.5$ UTA shown in the right panel, with $J=3 / 2$ lines appearing at higher densities and $J=1 / 2$ lines being prominent at low-density limit.

Future generations of X-ray spectrometers may be able to use such differences to measure the density of the absorbing gas. This would make it possible to deduce the location of the gas. Nevertheless, improved accuracy of the theoretical wavelengths would be needed for such a test to be definitive.

Absorption line spectroscopy generally has a degeneracy introduced by the fact that the ionization distribution determines an ionization parameter, the ratio of the flux of ionizing photons to gas density. Literature on this type of analysis is extensive, and summarized by Chakravorty et al. (2009). The ionization itself is not sensitive to either flux or density. By measuring the density and ionization parameter, the flux could be deduced, which then leads to the determination of the source-cloud separation.

Tests show that the density effects do not have a significant effect on the ionization of the gas because the total absorption oscillator strengths are similar. In the current implementation, we assume the low-density limit in computing the effects of UTA transitions and the resulting spectra.

The ground terms of $\mathrm{Fe}^{14+}$ and $\mathrm{Fe}^{15+}$ are simpler, having only one level, so such distinctions do not occur.

\subsection{Probing the Thermal Stability of the Warm Absorber}

The nature of the absorbing and emitting clouds in AGN is a long-standing problem (Osterbrock \& Ferland 2006; 

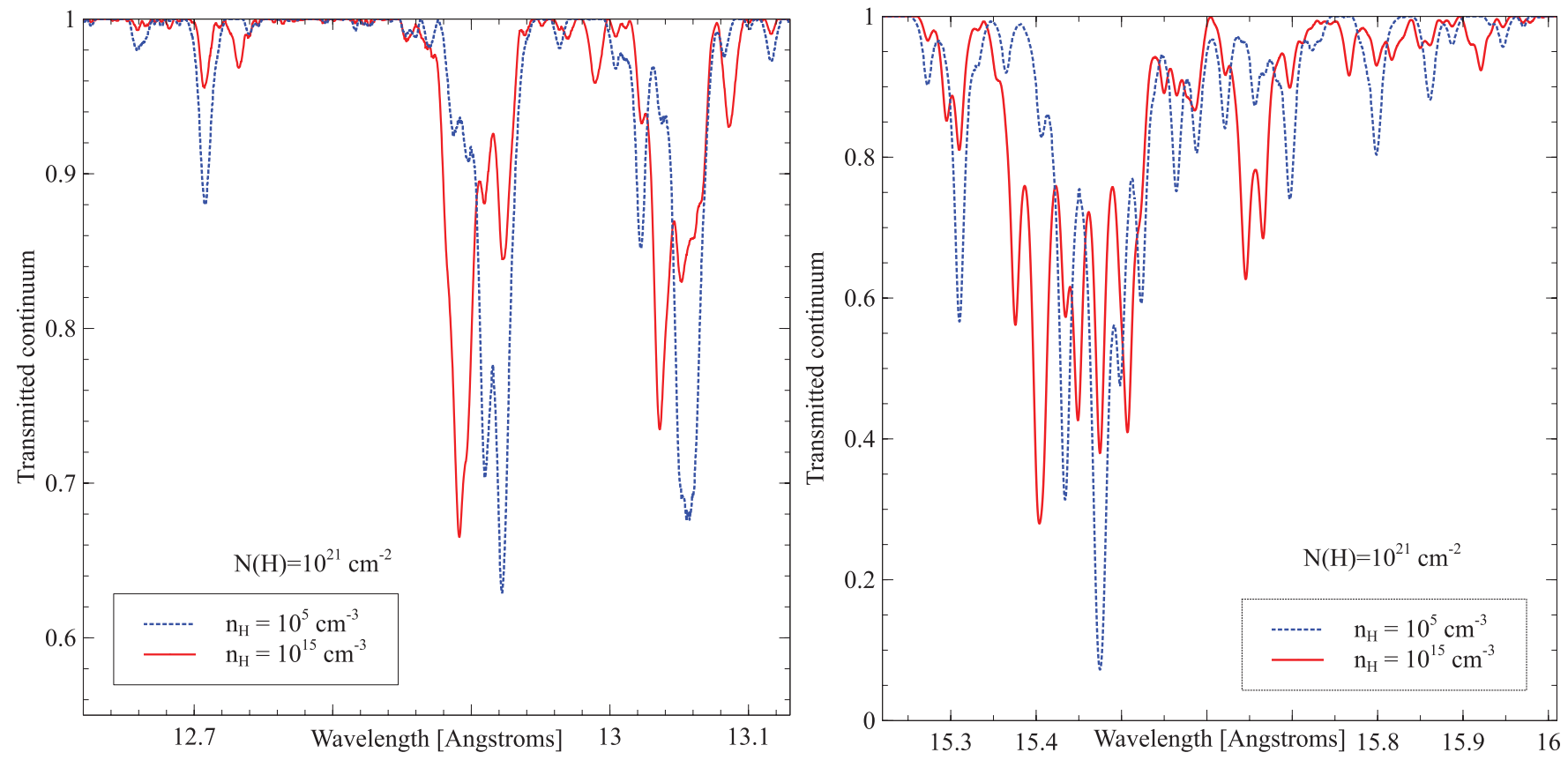

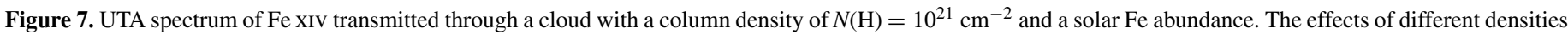

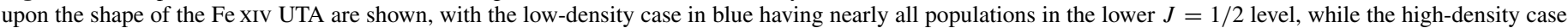
has level populations within the ground term proportional to their statistical weight. This calculation only included Fe XIV.

(A color version of this figure is available in the online journal.)

Chakravorty et al. 2009). By analogy with the local interstellar medium, several gas phases are thought to be present at one location, each in equilibrium with the radiation field, but having different levels of ionization and a range of temperatures, but the same gas pressure. This has been discussed extensively in the literature (Krolik et al. 1981; Hess et al. 1997; Krolik \& Kriss 2001; Komossa \& Mathur 2001; Reynolds \& Fabian 1995).

The ionization, temperature, and spectrum of clouds that can exist is determined by the type of stability analysis shown in Figure 8. This shows the familiar "S curve," which is computed for an optically thin cell of gas exposed to the AGN radiation field. Thermally stable phases have positive slope, while regions with negative slope are unstable so that gas will only exist in these regions for a short time. The shape of the $S$ curve determines the properties of clouds which are thermally stable and so live long enough to contribute to the observed spectrum.

Recent S-curve calculations have focused on how properties of the AGN might affect its shape, and so determine which clouds might exist. The literature on this topic is vast, and recent examples include Holczer et al. $(2007,2010)$ and Holczer \& Behar (2012), who examine observational determinations of the stable points on the S curve, Chakravorty et al. (2008), who show the effects of updated dielectronic recombination rate coefficients, Chakravorty et al. (2009), who do a systematic stability analysis, and Chakravorty et al. (2012), who discuss the influence of the spectral energy distribution (SED) on the cloud stability.

Here, we show the effects of the improvements discussed in this paper, using version C13 of Cloudy (Ferland et al. 2013). Some other recent improvements to the atomic data, which focus on the atomic models used to compute the cooling, are described by Lykins et al. (2013). Figure 8 shows the thermal stability curve resulting from the improved atomic physics in this paper. Many model parameters are taken from Chakravorty et al. (2009). The $x$-axis is the ratio of the dimensionless ionization

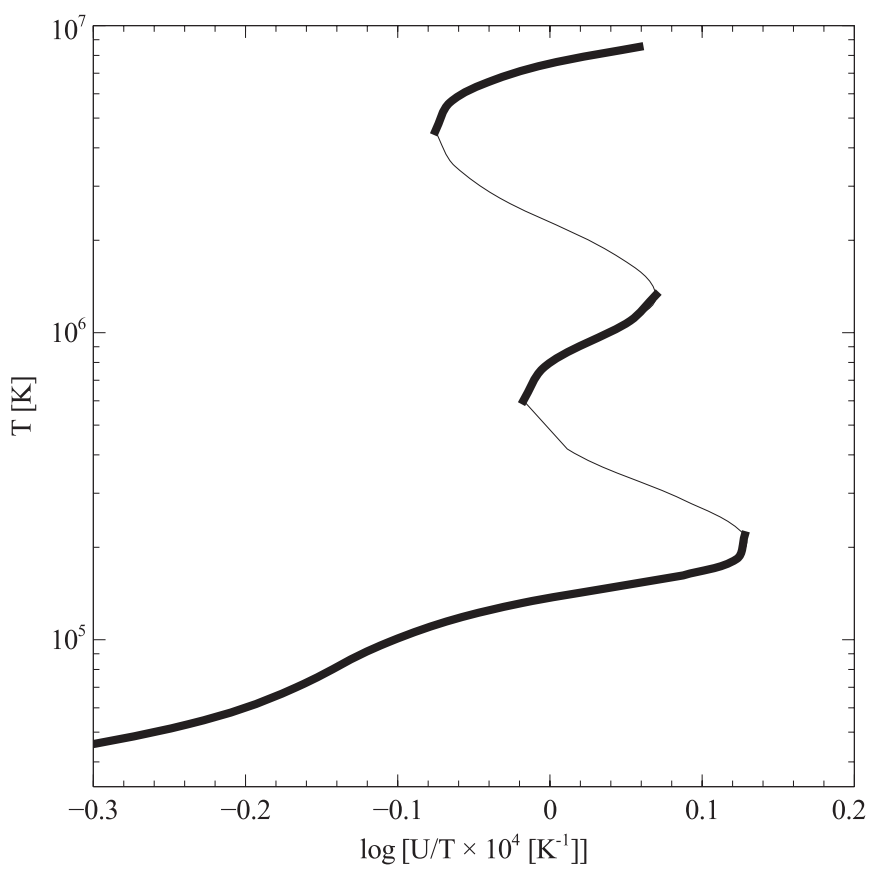

Figure 8. Newly computed AGN thermal stability curve. The default AGN SED described by Chakravorty et al. (2009) and a hydrogen density of $10^{8} \mathrm{~cm}^{-3}$ are assumed. Thermally stable regions are shown by the thicker lines.

parameter $U$, defined as the ratio of ionizing photon to hydrogen densities, to the gas kinetic temperature, while the latter is the $y$-axis. Thermally stable regions, those with positive slope, are shown as the heavier lines.

In a series of papers Holczer et al. (2007, 2010), and Holczer \& Behar (2012) infer from observations of column densities of certain ions that temperatures between $4.5<\log T<5.0$ are missing and ascribe it to thermal instability. On the other hand, 


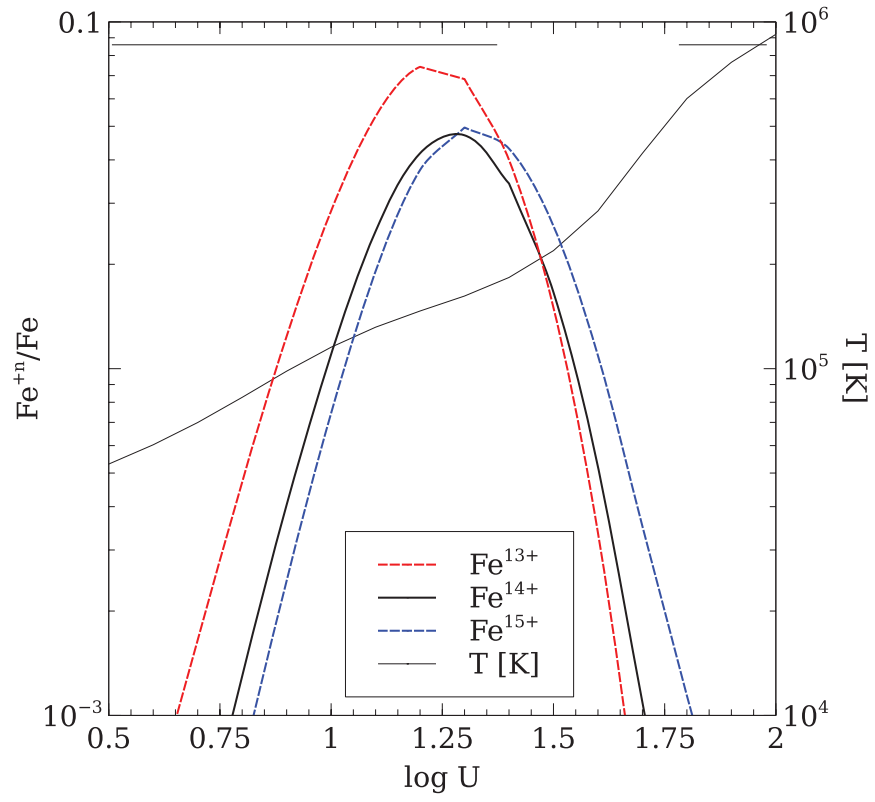

Figure 9. Fe ionization distribution across Figure 8, where the gas kinetic temperature is indicated by the solid line increasing from left to right. Horizontal lines at the top of the figure mark the thermally stable regions of Figure 8. The Fe ions considered in this paper probe the high temperature end of the cool branch in Figure 8, unstable regions, and the low temperature end of the middle stable branch.

(A color version of this figure is available in the online journal.)

this region is stable in the current calculation with its assumed parameters. The shape of the stability curve is affected by several other ingredients besides the atomic data. The composition assumed, and the form of the SED, also change it. This suggests that the presence or absence of stable gas could be used to infer the SED or gas composition, among other properties of the AGN.

Figure 9 shows the distribution of ionization stages of $\mathrm{Fe}$ as a function of the ionization parameter $U$. The ions discussed in this paper peak in the range $\log U \sim 1-2$. These probe the upper bound of the low- $T$ branch of thermally stable gas, the lowest unstable region, and the low- $T$ part of the middle stable branch. Horizontal lines in the upper part of Figure 9 indicate the regions where the gas is stable.

The atomic data presented in this paper affect the details of the transition between the cool and middle stable branches. Figure 10 shows the ratio of UTA to total ionization for several charge states of $\mathrm{Fe}$, where the filled circles include the data presented in this paper, while the crosses represent the older data. The larger number of lines derived here results in a larger UTA ionization rate, as shown in the figure. By UTA ionization we mean the effect of autoionization following innershell photoexcitation. UTAs have the greatest effect on the ions which occur around the transition from the cool to middle stable branch, and their physics affects the details of this transition.

Finally, in Figure 11 we show the spectrum produced by an intervening cloud near the upper range of the cool stable branch. This cloud has solar abundances, a column density of $N(\mathrm{H})=10^{21} \mathrm{~cm}^{-2}$, and an ionization parameter of $\log U=$ 1.25. A portion of the coarse continuum which is used for continuum radiative transfer and atomic rates is shown in the upper panel. The assumed SED, which includes the "Big Bump" from the central accretion disk and a non-thermal X-ray power law, has many absorption features superimposed. Emission lines

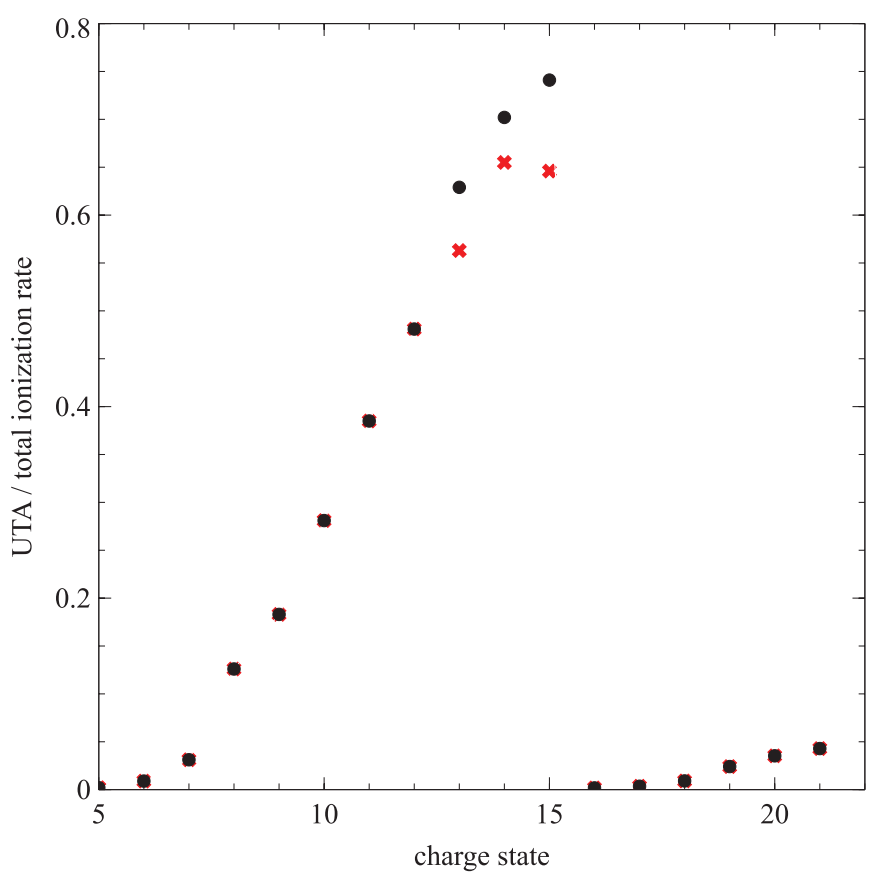

Figure 10. Ratio of the UTA ionization to total ionization rate for several $\mathrm{Fe}$ charge stages. Filled circles include the results presented in this paper, while the crosses represent the older data. These are for the conditions occurring across Figure 8 . Rates are evaluated at the illuminated face so line self-shielding is not important. UTA ionization is the dominant ionization process for the ions considered in this paper.

(A color version of this figure is available in the online journal.)
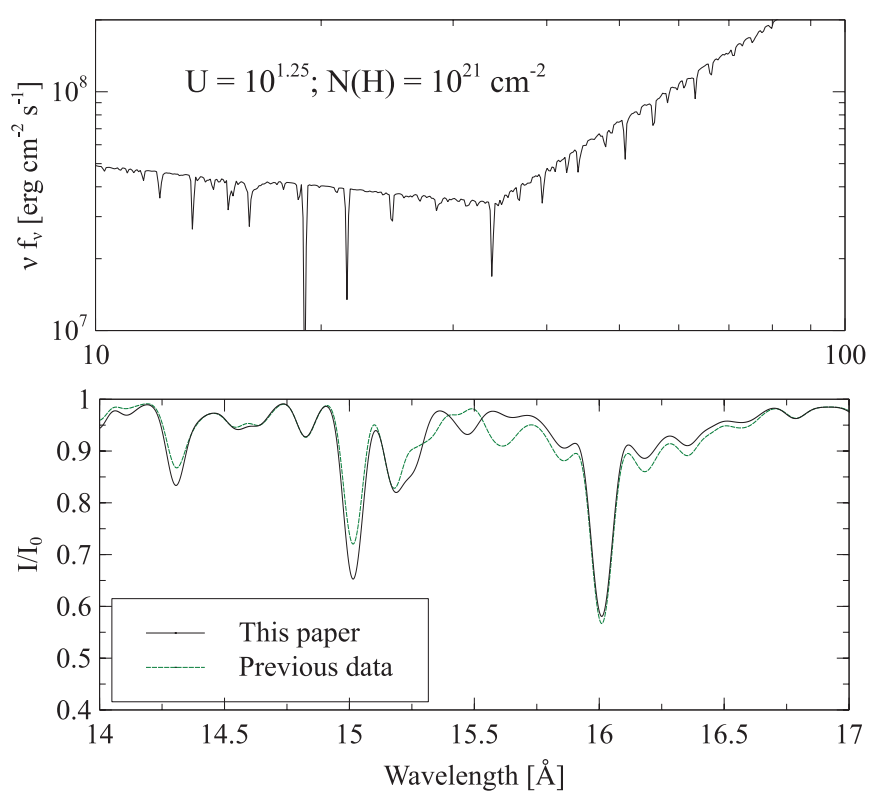

Figure 11. Spectrum of a cloud lying along the line of sight to an AGN. Cloud parameters are indicated in the upper panel, which shows the transmitted coarse continuum. The lower panel shows a small portion of the fine continuum with predictions using the atomic data summarized in this paper in black, and with older UTA line data in green.

(A color version of this figure is available in the online journal.)

are also produced by the cloud but are weak in this portion of the spectrum due to the low gas temperature.

The lower panel shows a small portion of the fine continuum in the neighborhood of the $\sim 15 \AA$ UTA feature. Two curves are shown, with the solid line using the results presented in this 
paper, and the dashed line employing the other data sources summarized above. Significant differences are present.

\section{CONCLUSIONS}

This paper has summarized advances in the atomic data needed to simulate conditions in a photoionized plasma. All of these improvements are now in the development version of the plasma simulation code Cloudy, and will be part of the next major release. The specific advances are the following.

1. We have calculated a large set of atomic data for UTA lines using the CI method implemented in the cIV 3 code. This new data set substantially extends previous atomic data of Kisielius et al. (2003) in two ways. First, the E1 transitions from the inner $2 s$ shell are determined. Second, the data sets for Fe XIV, Fe XV, and Fe XVI include lines $2 l-4 l, 5 l$ in addition to the earlier determined lines $2 p-3 l$.

2. We have incorporated this large set of new UTA data into the spectral simulation code Cloudy, and applied it to problems in AGN. These improvements will be part of the next major release of the code.

3. We summarize our data sources for UTA transitions. There are still missing data, even for very important ions. These should be a priority for new theoretical calculations.

4. We summarize how our data, which were computationally very expensive to undertake, compare to simpler calculations. Line wavelengths differ (although insignificantly) due to differences in the computed level energies, but the transition rates are in good agreement.

5. The UTA lines are often strongly damped, many having damping parameters $a \gg 1$. We have improved the form of the Voigt function used by Cloudy to handle such strongly damped lines.

6. We show how the Fe XIV UTA at $\lambda 15.5$ can be used to measure the density of the gas, or identify whether the density is significantly above or below $10^{9} \mathrm{~cm}^{-3}$. Such measurements would help determine the location of the warm absorber in AGN.

7. The total ionization rate is increased by roughly $30 \%$ with the new set of UTA data, which have far more lines. This changes the ionization of the gas and alters the thermal properties of photoionized gas exposed to a typical AGN SED.

8. We present a newly computed thermal stability "S curve" using the new data. We show that the Fe ions considered in this paper are produced in the warmer parts of the cool thermally stable branch, an unstable region, and in cooler parts of the middle stable branch. As a result, these lines probe the portions of the $\mathrm{S}$ curve which determine which cloud parameters can persist. Future work will investigate the effects changes in these regions have upon the observed spectrum.
G.J.F. acknowledges support by NSF (0908877; 1108928; and 1109061), NASA (10-ATP10-0053, 10-ADAP10-0073, and NNX12AH73G), JPL (RSA No 1430426), and STScI (HST-AR-12125.01, GO-12560, and HST-GO-12309). R.K. acknowledges support from the project VP1-3.1-ŠMM-07-K02-013 funded by European Social Fund under the Global Grant measure. F.P.K. is grateful to AWE Aldermaston for the award of a William Penney Fellowship. P.v.H. acknowledges support from the Belgian Science Policy office through the ESA PRODEX programme.

\section{REFERENCES}

Badnell, N. R., Bautista, M. A., Butler, D., et al. 2005, MNRAS, 360, 458 Bar-Shalom, A., Klapisch, M., \& Oreg, J. 2001, JQSRT, 71, 169

Behar, E., Sako, M., \& Kahn, S. M. 2001, ApJ, 563, 497

Beiersdorfer, P., Diaz, F., \& Ishikawa, Y. 2012, ApJ, 745, 167

Brown, G. V., Beiersdirfer, P., Chen, H., Chen, M. H., \& Reed, K. J. 2001, ApJL, 557, L75

Chakravorty, S., Kembhavi, A. K., Elvis, M., \& Ferland, G. 2009, MNRAS, 393, 83

Chakravorty, S., Kembhavi, A. K., Elvis, M., Ferland, G., \& Badnell, N. R. 2008, MNRAS, 384, L24

Chakravorty, S., Misra, R., Elvis, M., Kembhavi, A. K., \& Ferland, G. 2012, MNRAS, 422, 637

Ferland, G. J., Porter, R. L., van Hoof, P. A. M., et al. 2013, RMxAA, 49, 137

Gu, M. F., Holczer, T., Behar, E., \& Kahn, S. M. 2006, ApJ, 641, 1227

Hess, C. J., Kahn, S. M., \& Paerels, F. B. S. 1997, ApJ, 478, 94

Hibbert, A. 1975, CoPhC, 9, 141

Hibbert, A., Glass, R., \& Froese Fischer, C. 1991, CoPhC, 64, 455

Holczer, T., \& Behar, E. 2012, ApJ, 747, 71

Holczer, T., Behar, E., \& Arav, N. 2010, ApJ, 708, 981

Holczer, T., Behar, E., \& Kaspi, S. 2007, ApJ, 663, 799

Kisielius, R., Hibbert, A., Ferland, G. J., et al. 2003, MNRAS, 344, 696

Komossa, S., \& Mathur, S. 2001, A\&A, 374, 914

Krolik, J. H., \& Kriss, G. A. 2001, ApJ, 561, 684

Krolik, J. H., McKee, C. F., \& Tarter, C. B. 1981, ApJ, 249, 422

Lestinsky, M., Badnell, N. R., Bernhardt, D., et al. 2009, ApJ, 698, 648

Lindgren, I. 1974, JPhB, 7, 2441

Longinotti, A. L., Costantini, E., Petrucci, P. O., et al. 2010, A\&A, 510, A92

Lykins, M. L., Ferland, G. J., Porter, R. L., et al. 2013, MNRAS, 429, 3133

Mihalas, D. 1978, Stellar Atmospheres (2nd edition; San Francisco, CA: Freeman)

Osterbrock, D., \& Ferland, G. J. (ed.) 2006, Astrophysics of Gaseous Nebulae and Active Galactic Nuclei (2nd edition; Sausalito, CA: University Science Books)

Reynolds, C. S., \& Fabian, A. C. 1995, MNRAS, 273, 1167

Rutten, R. J. 2003, Utrecht University lecture notes, 8th edition. Available from http://www.astro.uu.nl/ rutten

Sako, M., Kahn, S. M., Behar, E., et al. 2001, A\&A, 365, 168

Shaw, G., Ferland, G. J., Abel, N. P., Stancil, P. C., \& van Hoof, P. A. M. 2005, ApJ, 624, 794

Simon, M. C., Crespo Lpez-Urrutia, J. R., Beilmann, C., et al. 2010, PhRvL, 105,183001

Wells, R. J. 1999, JQSRT, 62, 29

Zaghloul, M. R., \& Ali, A. N. 2011, ACM Trans. Math. Softw., 38, 15 